\title{
Shot noise for entangled and spin-polarized electrons
}

\author{
J. C. Egues*, P. Recher, D. S. Saraga, V. N. Golovach, G. Burkard, E. V. \\ Sukhorukov, and D. Loss \\ Department of Physics and Astronomy, University of Basel, Klingelbergstrasse 82, CH-4056 \\ Basel, Switzerland
}

\begin{abstract}
We review our recent contributions on shot noise for entangled electrons and spin-polarized currents in novel mesoscopic geometries. We first discuss some of our recent proposals for electron entanglers involving a superconductor coupled to a double dot in the Coulomb blockade regime, a superconductor tunnel-coupled to Luttinger-liquid leads, and a triple-dot setup coupled to Fermi leads. We briefly survey some of the available possibilities for spin-polarized sources. We use the scattering approach to calculate current and shot noise for spin-polarized currents and entangled/unentangled electron pairs in a novel beamsplitter geometry with a local Rashba spin-orbit (s-o) interaction in the incoming leads. For single-moded incoming leads, we find continuous bunching and antibunching behaviors for the entangled pairs - triplet and singlet - as a function of the Rashba rotation angle. In addition, we find that unentangled triplets and the entangled one exhibit distinct shot noise; this should allow their identification via noise measurements. Shot noise for spin-polarized currents shows sizable oscillations as a function of the Rashba phase. This happens only for electrons injected perpendicular to the Rashba rotation axis; spin-polarized carriers along the Rashba axis are noiseless. The Rashba coupling constant $\alpha$ is directly related to the Fano factor and could be extracted via noise measurements. For incoming leads with s-o induced interband-coupled channels, we find an additional spin rotation for electrons with energies near the crossing of the bands where interband coupling is relevant. This gives rise to an additional modulation of the noise for both electron pairs and spin-polarized currents. Finally, we briefly discuss shot noise for a double dot near the Kondo regime.
\end{abstract}

Keywords: Shot noise, entanglement, spintronics, quantum dots, Luttinger liquids

\section{Introduction}

Fluctuations of the current away from its average usually contain supplementary information, not provided by average-current measurements alone. This is particularly true in the non-linear response regime where these quantities are not related via the fluctuation-dissipation theorem. At zero temperature, non-equilibrium current noise is due to the discreteness of the electron charge and is termed shot noise. This dynamic noise was first investigated by Schottky in connection with thermionic emission [1]. Quantum shot noise has reached its come of age in the past decade or so and constitutes now an indispensable tool to probe mesoscopic transport [2]; in particular, the role of fundamental correlations such as those imposed by quantum statistics.

\footnotetext{
* Permanent address: Department of Physics and Informatics, University of São Paulo at São Carlos, 13560-970 São Carlos/SP, Brazil.
}

(C) 2018 Kluwer Academic Publishers. Printed in the Netherlands. 
More recently, shot noise has been investigated in connection with transport of entangled [3]-[8] and spin polarized electrons [4], [8]-[11] and has proved to be a useful probe for both entanglement and spin-polarized transport. Entanglement [12] is perhaps one of the most intriguing features of quantum mechanics since it involves the concept of non-locality. Two-particle entanglement is the simplest conceivable form of entanglement. Yet, these Einstein-Podolsky-Rosen (EPR) pairs play a fundamental role in potentially revolutionary implementations of quantum computation, communication, and information processing [13]. In this context, such a pair represents two qubits in an entangled state. The generation and detection of EPR pairs of photons has already been accomplished. On the other hand, research involving two-particle entanglement of massive particles (e.g. electrons) in a solidstate matrix is still in its infancy, with a few proposals for its physical implementation; some of these involve quantum-dot setups as sources of mobile spin-entangled electrons [4], [14], [15]. Spin-polarized transport [16], [17], [18], on the other hand, is a crucial ingredient in semiconductor spintronics where the spin (and/or possibly the charge) of the carriers play the relevant role in a device operation. To date, robust spin injection has been achieved in Mn-based semiconductor layers (pin diode structures) [16]. Highefficiency spin injection in other semiconductor systems such as hybrid ferromagnetic/semiconductor junctions is still challenging.

It is clear that the ability to create, transport, coherently manipulate, and detect entangled electrons and spin-polarized currents in mesoscopic systems is highly desirable. Here we review some of our recent works [4], [7], [8], [14], [15], [19], [82] addressing some of these issues and others in connection with noise. Shot noise provides an additional probe in these novel transport settings. We first address the production of mobile entangled electron pairs (Sec. 2). We discuss three proposals involving a superconductor coupled to two dots [14], a superconductor coupled to Luttinger-liquid leads [19], and a triple-dot arrangement [15]. Our detailed analysis of these "entanglers" does not reveal any intrinsic limitation to their experimental feasibility. We also mention some of the available sources of spin-polarized electrons (Sec. 3). Ballistic spin filtering with spin-selective semimagnetic tunnel barriers [17] and quantum dots as spin filters [18] are also briefly discussed.

We investigate transport of entangled and spin polarized electrons in a beamsplitter (four-port) configuration [21], [22] with a local Rashba spin-orbit interaction in the incoming leads [23], Fig. (1). A local Rashba term provides a convenient way to coherently spin-rotate electrons as they traverse quasi one-dimensional channels, as was first pointed out by Datta and Das [24]. Within the scattering formalism [2], we calculate shot noise for both entangled and spin-polarized electrons.

For entangled electrons, shot noise is particularly relevant as a probe for fundamental two-particle interference. More specifically, shot noise (charge 


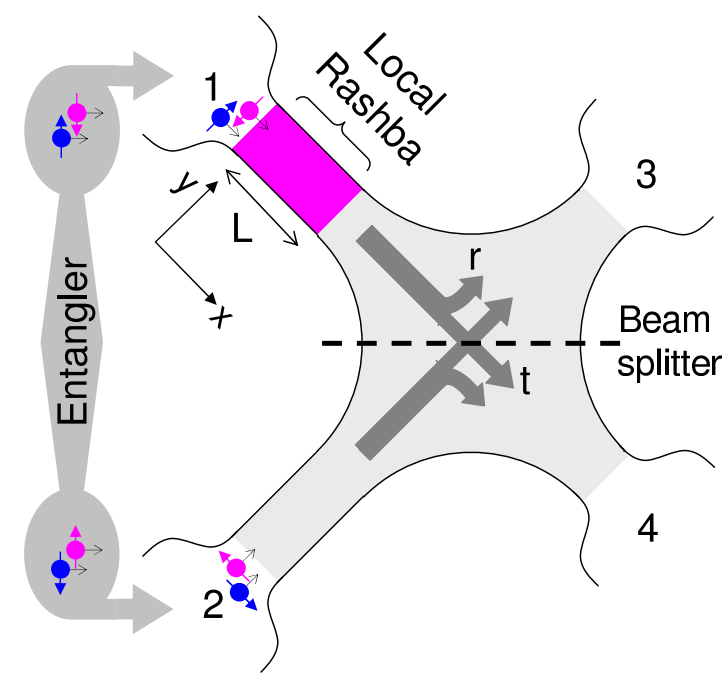

Figure 1. Novel electron beam-splitter geometry with a local Rashba s-o interaction in lead 1. An entangler or a spin-polarized electron source (not shown) inject either entangled pairs or spin-polarized carriers into leads 1 and 2. The portion of the entangled pairs (or the spin-polarized carriers) traversing lead 1 undergoes a Rashba-induced spin rotation. This continuously changes the symmetry of the spin part of the pair wave function. Adapted from Ref. [8].

noise) directly probes the orbital symmetry of the EPR pair wave function. However, the symmetry of the orbital degree of freedom ("the charge") is intrinsically tied to that of the spin part of the pair wave function via the Pauli principle. That is, the total electron-pair wave function is antisymmetric thus imposing a fundamental connection between the spin and orbital parts of the pair wave function. Hence charge noise measurements probe in fact the spin symmetry of the pair. Moreover, if one can alter the spin state of the pair (say, via some proper coherent spin rotation) this will definitely influence shot-noise measurements. This is precisely what we find here for singlet and triplet pairs.

The coherent local Rashba spin rotation in one of the incoming leads of our setup, continuously alters the (spin) symmetry of the pair wave function thus giving rise to sizable shot noise oscillations as a function of the Rashba phase. Noise measurements in our novel beam-splitter should allow one to distinguish entangled triplets from singlets and entangled triplets from the unentangled ones, through their Rashba phase. Entangled pairs display continuous bunching/antibunching behavior. In addition, triplets (entangled 
or not) defined along different quantization axes $(x, y$, or $z)$ exhibit distinctive noise, thus allowing the detection of their spin polarization via charge noise measurements.

Shot noise for spin-polarized currents also probes effects imposed by the Pauli principle through the Fermi functions in the leads. These currents also exhibit Rashba-induced oscillations for spin polarizations perpendicular to the Rashba rotation axis. We find zero shot noise for spin-polarized carriers with polarizations along the Rashba axis and for unpolarized injection. Moreover, the Rashba-induced modulations of the Fano factor for both entangled and spin-polarized electrons offer a direct way to extract the s-o coupling constant via noise measurements.

We also consider incoming leads with two transverse channels. In the presence of a weak s-o induced interchannel coupling, we find an additional spin rotation due to the coherent transfer of carriers between the coupled channels in lead 1. This extra rotation gives rise to further modulation of the shot noise characteristics for both entangled and spin-polarized currents; this happens only for carriers with energies near the band crossings in lead 1. Finally, we briefly discuss shot noise for transport through a double dot near the Kondo regime [82].

\section{Sources of mobile spin-entangled electrons}

A challenge in mesoscopic physics is the experimental realization of an electron "entangler" - a device creating mobile entangled electrons which are spatially separated. Indeed, these are essential for quantum communication schemes and experimental tests of quantum non-locality with massive particles. First, one should note that entanglement is rather the rule than the exception in nature, as it arises naturally from Fermi statistics. For instance, the ground state of a helium atom is the spin singlet $|\uparrow \downarrow\rangle-|\downarrow \uparrow\rangle$. Similarly, one finds a singlet in the ground state of a quantum dot with two electrons. These "artificial atoms" [25] are very attractive for manipulations at the single electron level, as they possess tunable parameters and allow coupling to mesoscopic leads - contrary to real atoms. However, such "local" entangled singlets are not readily useful for quantum computation and communication, as these require control over each individual electron as well as non-local correlations. An improvement in this direction is given by two coupled quantum dots with a single electron in each dot [26], where the spin-entangled electrons are already spatially separated by strong on-site Coulomb repulsion (like in a hydrogen molecule). In this setup, one could create mobile entangled electrons by simultaneously lowering the tunnel barriers coupling each dot to separate leads. Another natural source of spin entanglement can be found in superconductors, as these contain Cooper pairs with singlet spin 
wave functions. It was first shown in Ref. [27] how a non-local entangled state is created in two uncoupled quantum dots when coupled to the same superconductor. In a non-equilibrium situation, the Cooper pairs can be extracted to normal leads by Andreev tunnelling, thus creating a flow of entangled pairs [14],[19],[28]-[31].

A crucial requirement for an entangler is to create spatially separated entangled electrons; hence one must avoid whole entangled pairs entering the same lead. As will be shown below, energy conservation is an efficient mechanism for the suppression of undesired channels. For this, interactions can play a decisive role. For instance, one can use Coulomb repulsion in quantum dots [14],[15] or in Luttinger liquids [19],[28]. Finally, we mention recent entangler proposals using leads with narrow bandwidth [32] and/or generic quantum interference effects [33]. In the following, we discuss our proposals towards the realization of an entangler that produces mobile non-local singlets [34]. We set $\hbar=1$ in this section.

\subsection{SUPERCONDUCTOR-BASED ELECTRON ENTANGLERS}

Here we envision a non-equilibrium situation in which the electrons of a Cooper pair tunnel coherently by means of an Andreev tunnelling event from a SC to two separate normal leads, one electron per lead. Due to an applied bias voltage, the electron pairs can move into the leads thus giving rise to mobile spin entanglement. Note that an (unentangled) single-particle current is strongly suppressed by energy conservation as long as both the temperature and the bias are much smaller than the superconducting gap. In the following we review two proposals where we exploit the repulsive Coulomb charging energy between the two spin-entangled electrons in order to separate them so that the residual current in the leads is carried by non-local singlets. We

show that such entanglers meet all requirements for subsequent detection of spin-entangled electrons via noise measurements (charge measurement, see Secs. 5 and 8) or via spin-projection measurements (Bell-type measurement, see Sec. 3.3).

\subsubsection{Andreev entangler with quantum dots}

The proposed entangler setup (see Fig. 2) consists of a SC with chemical potential $\mu_{S}$ which is weakly coupled to two quantum dots (QDs) in the Coulomb blockade regime [25]. These QDs are in turn weakly coupled to outgoing Fermi liquid leads, held at the same chemical potential $\mu_{l}$. A bias voltage $\Delta \mu=\mu_{S}-\mu_{l}$ is applied between the $\mathrm{SC}$ and the leads. The tunnelling amplitudes between the SC and the dots, and dots and leads, are denoted by $T_{S D}$ and $T_{D L}$, respectively (see Fig. 2). The two intermediate QDs in the Coulomb blockade regime have chemical potentials $\epsilon_{1}$ and $\epsilon_{2}$, respectively. These can be tuned via external gate voltages, such that the tunnelling of two 


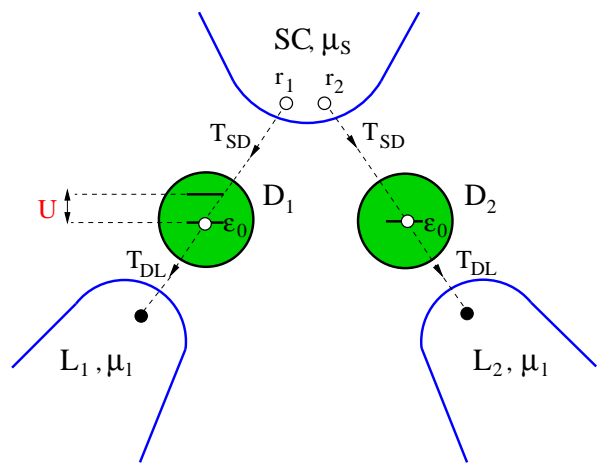

Figure 2. The entangler setup. Two spin-entangled electrons forming a Cooper pair tunnel with amplitude $T_{S D}$ from points $\mathbf{r}_{1}$ and $\mathbf{r}_{2}$ of the superconductor, SC, to two dots, $D_{1}$ and $D_{2}$, by means of Andreev tunnelling. The dots are tunnel-coupled to normal Fermi liquid leads $\mathrm{L}_{1}$ and $\mathrm{L}_{2}$, with tunnelling amplitude $T_{D L}$. The superconductor and leads are kept at chemical potentials $\mu_{S}$ and $\mu_{l}$, respectively. Adapted from [14].

electrons via different dots into different leads is resonant for $\epsilon_{1}+\epsilon_{2}=2 \mu_{S}$ [35]. As it turns out [14], this two-particle resonance is suppressed for the tunnelling of two electrons via the same dot into the same lead by the on-site repulsion $U$ of the dots and/or the superconducting gap $\Delta$. Next, we specify the parameter regime of interest here in which the initial spin-entanglement of a Cooper pair in the SC is successfully transported to the leads.

Besides the fact that single-electron tunnelling and tunnelling of two electrons via the same dot should be excluded, we also have to suppress transport of electrons which are already on the QDs. This could lead to effective spin-flips on the QDs, which would destroy the spin entanglement of the two electrons tunnelling into the Fermi leads. A further source of unwanted spin-flips on the QDs is provided by its coupling to the Fermi liquid leads via particle-hole excitations in the leads. The QDs can be treated each as one localized spindegenerate level as long as the mean level spacing $\delta \epsilon$ of the dots exceeds both the bias voltage $\Delta \mu$ and the temperature $k_{B} T$. In addition, we require that each QD contains an even number of electrons with a spin-singlet ground state. A more detailed analysis of such a parameter regime is given in [14] and is stated here

$$
\Delta, U, \delta \epsilon>\Delta \mu>\gamma_{l}, k_{B} T \text {, and } \gamma_{l}>\gamma_{S} .
$$

In (1) the rates for tunnelling of an electron from the SC to the QDs and from the QDs to the Fermi leads are given by $\gamma_{S}=2 \pi \nu_{S}\left|T_{S D}\right|^{2}$ and $\gamma_{l}=$ $2 \pi \nu_{l}\left|T_{D L}\right|^{2}$, respectively, with $\nu_{S}$ and $\nu_{l}$ being the corresponding electron density of states per spin at the Fermi level. We consider asymmetric barriers $\gamma_{l}>\gamma_{s}$ in order to exclude correlations between subsequent Cooper pairs on the QDs. We work at the particular interesting resonance $\epsilon_{1}, \epsilon_{2} \simeq \mu_{S}$, where 
the injection of the electrons into different leads takes place at the same orbital energy. This is a crucial requirement for the subsequent detection of entanglement via noise $[4,8]$. In this regime, we have calculated and compared the stationary charge current of two spin-entangled electrons for two competing transport channels in a T-matrix approach.

The ratio of the desired current for two electrons tunnelling into different leads $\left(I_{1}\right)$ to the unwanted current for two electrons into the same lead $\left(I_{2}\right)$ is $[14]$

$$
\frac{I_{1}}{I_{2}}=\frac{4 \mathcal{E}^{2}}{\gamma^{2}}\left[\frac{\sin \left(k_{F} \delta r\right)}{k_{F} \delta r}\right]^{2} e^{-2 \delta r / \pi \xi}, \quad \frac{1}{\mathcal{E}}=\frac{1}{\pi \Delta}+\frac{1}{U},
$$

where $\gamma=\gamma_{1}+\gamma_{2}$. The current $I_{1}$ becomes exponentially suppressed with increasing distance $\delta r=\left|\mathbf{r}_{1}-\mathbf{r}_{2}\right|$ between the tunnelling points on the SC, on a scale given by the superconducting coherence length $\xi$ which is the size of a Cooper pair. This does not pose a severe restriction for conventional swave materials with $\xi$ typically being on the order of $\mu \mathrm{m}$. In the relevant case $\delta r<\xi$ the suppression is only polynomial $\propto 1 /\left(k_{F} \delta r\right)^{2}$, with $k_{F}$ being the Fermi wave vector in the SC. On the other hand, we see that the effect of the QDs consists in the suppression factor $(\gamma / \mathcal{E})^{2}$ for tunnelling into the same lead [36]. Thus, in addition to Eq. (1) we have to impose the condition $k_{F} \delta r<\mathcal{E} / \gamma$, which can be satisfied for small dots with $\mathcal{E} / \gamma \sim 100$ and $k_{F}^{-1} \sim 1 \AA$. As an experimental probe to test if the two spin-entangled electrons indeed separate and tunnel to different leads we suggest to join the two leads 1 and 2 to form an Aharonov-Bohm loop. In such a setup the different tunnelling paths of an Andreev process from the SC via the dots to the leads can interfere. As a result, the measured current as a function of the applied magnetic flux $\phi$ threading the loop contains a phase coherent part $I_{A B}$ which consists of oscillations with periods $h / e$ and $h / 2 e$ [14]

$$
I_{A B} \sim \sqrt{8 I_{1} I_{2}} \cos \left(\phi / \phi_{0}\right)+I_{2} \cos \left(2 \phi / \phi_{0}\right),
$$

with $\phi_{0}=h / e$ being the single-electron flux quantum. The ratio of the two contributions scales like $\sqrt{I_{1} / I_{2}}$ which suggest that by decreasing $I_{2}$ (e.g. by increasing $U$ ) the $h / 2 e$ oscillations should vanish faster than the $h / e$ ones.

We note that the efficiency as well as the absolute rate for the desired injection of two electrons into different leads can even be enhanced by using lower dimensional SCs $[19,37]$. In two dimensions (2D) we find that $I_{1} \propto 1 / k_{F} \delta r$ for large $k_{F} \delta r$, and in one dimension (1D) there is no suppression of the current and only an oscillatory behavior in $k_{F} \delta r$ is found. A 2D-SC can be realized by using a SC on top of a two-dimensional electron gas (2DEG) [38], where superconducting correlations are induced via the proximity effect in the 2DEG. In 1D, superconductivity was found in ropes of single-walled carbon nanotubes [39]. 
Finally, we note that the coherent injection of Cooper pairs by an Andreev process allows the detection of individual spin-entangled electron pairs in the leads. The delay time $\tau_{\text {delay }}$ between the two electrons of a pair is given by $1 / \Delta$, whereas the separation in time of subsequent pairs is given approximately by $\tau_{\text {pairs }} \sim 2 e / I_{1} \sim \gamma_{l} / \gamma_{S}^{2}$ (up to geometrical factors) [14]. For $\gamma_{S} \sim \gamma_{l} / 10 \sim 1 \mu \mathrm{eV}$ and $\Delta \sim 1 \mathrm{meV}$ we obtain that the delay time $\tau_{\text {delay }} \sim 1 / \Delta \sim 1$ ps is much smaller than the delivery time $\tau_{\text {pairs }}$ per entangled pair $2 e / I_{1} \sim 40 \mathrm{~ns}$. Such a time separation is indeed necessary in order to detect individual pairs of spin-entangled electrons.

\subsubsection{Andreev entangler with Luttinger-liquid leads}

Next we discuss a setup with an s-wave SC weakly coupled to the center (bulk) of two separate one-dimensional leads (quantum wires) 1,2 (see Fig. 3) which exhibit Luttinger liquid (LL) behavior, such as carbon nanotubes [40, $41,42]$. The leads are assumed to be infinitely extended and are described by conventional LL-theory [44].

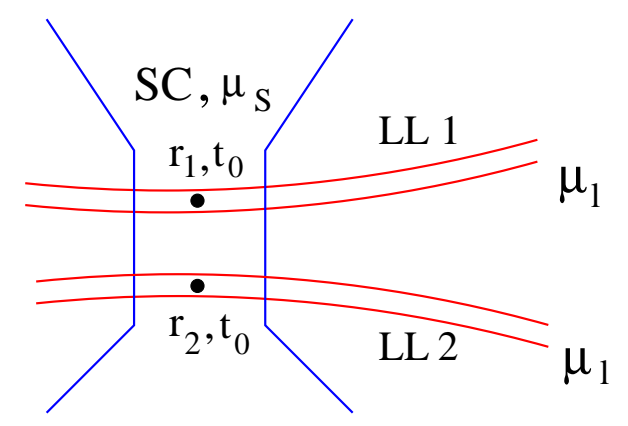

Figure 3. Two quantum wires 1,2, with chemical potential $\mu_{l}$ and described as infinitely long Luttinger liquids (LLs), are deposited on top of an s-wave superconductor (SC) with chemical potential $\mu_{S}$. The electrons of a Cooper pair can tunnel by means of an Andreev process from two points $\mathbf{r}_{1}$ and $\mathbf{r}_{2}$ on the SC to the center (bulk) of the two quantum wires 1 and 2, respectively, with tunnelling amplitude $t_{0}$. Adapted from [19].

Interacting electrons in one dimension lack the existence of quasi particles like they exist in a Fermi liquid and instead the low energy excitations are collective charge and spin modes. In the absence of backscattering interaction the velocities of the charge and spin excitations are given by $u_{\rho}=v_{F} / K_{\rho}$ for the charge and $u_{\sigma}=v_{F}$ for the spin, where $v_{F}$ is the Fermi velocity and $K_{\rho}<1$ for repulsive interaction between electrons $\left(K_{\rho}=1\right.$ corresponds to a 1D-Fermi gas). As a consequence of this non-Fermi liquid behavior, tunnelling into a LL is strongly suppressed at low energies. Therefore one should expect additional interaction effects in a two-particle tunnelling event (Andreev process) of a Cooper pair from the SC to the leads. We find that strong LL-correlations result in an additional suppression for tunnelling of 
two coherent electrons into the same LL compared to single electron tunnelling into a LL if the applied bias voltage $\mu$ between the SC and the two leads is much smaller than the energy gap $\Delta$ of the SC.

To quantify the effectiveness of such an entangler, we calculate the current for the two competing processes of tunnelling into different leads $\left(I_{1}\right)$ and into the same lead $\left(I_{2}\right)$ in lowest order via a tunnelling Hamiltonian approach. Again we account for a finite distance separation $\delta r$ between the two exit points on the SC when the two electrons of a Cooper pair tunnel to different leads. For the current $I_{1}$ of the desired pair-split process we obtain, in leading order in $\mu / \Delta$ and at zero temperature $[19,37]$

$$
I_{1}=\frac{I_{1}^{0}}{\Gamma\left(2 \gamma_{\rho}+2\right)} \frac{v_{F}}{u_{\rho}}\left[\frac{2 \Lambda \mu}{u_{\rho}}\right]^{2 \gamma_{\rho}}, I_{1}^{0}=\pi e \gamma^{2} \mu F_{d}[\delta r]
$$

where $\Gamma(x)$ is the Gamma function and $\Lambda$ is a short distance cut-off on the order of the lattice spacing in the LL and $\gamma=4 \pi \nu_{S} \nu_{l}\left|t_{0}\right|^{2}$ is the dimensionless tunnel conductance per spin with $t_{0}$ being the bare tunnelling amplitude for electrons to tunnel from the SC to the LL-leads (see Fig. 3). The electron density of states per spin at the Fermi level for the SC and the LL-leads are denoted by $\nu_{S}$ and $\nu_{l}$, respectively. The current $I_{1}$ has its characteristic non-linear form $I_{1} \propto \mu^{2 \gamma_{\rho}+1}$ with $\gamma_{\rho}=\left(K_{\rho}+K_{\rho}^{-1}\right) / 4-1 / 2>0$ being the exponent for tunnelling into the bulk of a single LL. The factor $F_{d}[\delta r]$ in (4) depends on the geometry of the device and is given here again by $F_{d}[\delta r]=\left[\sin \left(k_{F} \delta r\right) / k_{F} \delta r\right]^{2} \exp (-2 \delta r / \pi \xi)$ for the case of a 3D-SC. In complete analogy to subsection 2.1.1 the power law suppression in $k_{F} \delta r$ gets weaker in lower dimensions.

This result should be compared with the unwanted transport channel where two electrons of a Cooper pair tunnel into the same lead 1 or 2 but with $\delta r=0$. We find that such processes are indeed suppressed by strong LLcorrelations if $\mu<\Delta$. The result for the current ratio $I_{2} / I_{1}$ in leading order in $\mu / \Delta$ and for zero temperature is $[19,37]$

$$
\frac{I_{2}}{I_{1}}=F_{d}^{-1}[\delta r] \sum_{b= \pm 1} A_{b}\left(\frac{2 \mu}{\Delta}\right)^{2 \gamma_{\rho b}}, \gamma_{\rho+}=\gamma_{\rho}, \gamma_{\rho-}=\gamma_{\rho}+\left(1-K_{\rho}\right) / 2
$$

where $A_{b}$ is an interaction dependent constant [45]. The result (5) shows that the current $I_{2}$ for injection of two electrons into the same lead is suppressed compared to $I_{1}$ by a factor of $(2 \mu / \Delta)^{2 \gamma_{\rho+}}$, if both electrons are injected into the same branch (left or right movers), or by $(2 \mu / \Delta)^{2 \gamma_{\rho-}}$ if the two electrons travel in different directions [46]. The suppression of the current $I_{2}$ by $1 / \Delta$ reflects the two-particle correlation effect in the LL, when the electrons tunnel into the same lead. The larger $\Delta$, the shorter the delay time is between the arrivals of the two partner electrons of a Cooper pair, and, in 
turn, the more the second electron tunnelling into the same lead will feel the existence of the first one which is already present in the LL. This behavior is similar to the Coulomb blockade effect in QDs, see subsection 2.1.1. Concrete realizations of LL-behavior is found in metallic carbon nanotubes with similar exponents as derived here [41, 42]. In metallic single-walled carbon nanotubes $K_{\rho} \sim 0.2$ [40] which corresponds to $2 \gamma_{\rho} \sim 1.6$. This suggests the rough estimate $(2 \mu / \Delta)<1 / k_{F} \delta r$ for the entangler to be efficient. As a consequence, voltages in the range $k_{B} T<\mu<100 \mu \mathrm{eV}$ are required for $\delta r \sim \mathrm{nm}$ and $\Delta \sim 1 \mathrm{meV}$. In addition, nanotubes were reported to be very good spin conductors [43] with estimated spin-flip scattering lengths of the order of $\mu \mathrm{m}$ [28].

We remark that in order to use the beam-splitter setup to detect spin-entanglement via noise the two LL-leads can be coupled further to Fermi liquid leads. In such a setup the LL-leads then would act as QDs [47]. Another way to prove spin-entanglement is to carry out spin-dependent current-current correlation measurements between the two LLs. Such spin dependent currents can be measured e.g. via spin filters (Sec. 3).

\subsection{TRIPLE-QUANTUM DOT ENTANGLER}

In this proposal [15], the pair of spin-entangled electrons is provided by the ground state of a single quantum dot $D_{C}$ with an even number of electrons, which is the spin-singlet [48]; see Fig. 4. In the Coulomb blockade regime [25], electron interactions in each dot create a large charging energy $U$ that provides the energy filtering necessary for the suppression of the nonentangled currents. These arise either from the escape of the pair to the same lead, or from the transport of a single electron. The idea is to create a resonance for the joint transport of the two electrons from $D_{C}$ to secondary
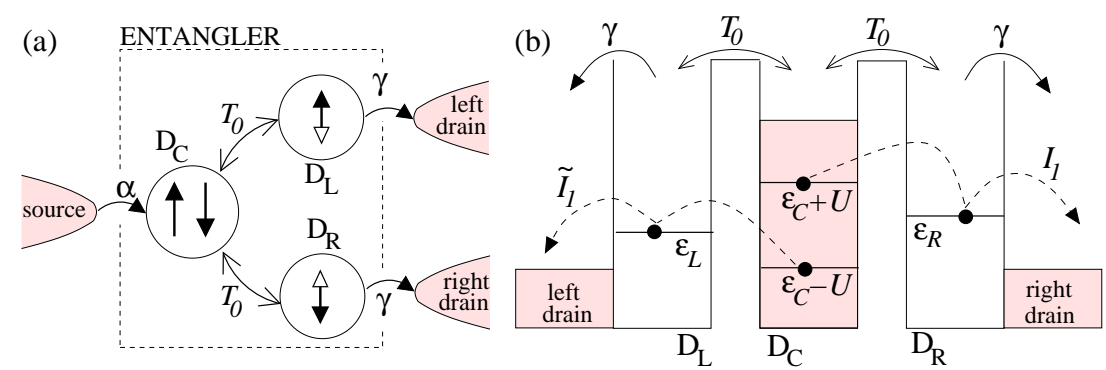

Figure 4. (a) Setup of the triple quantum dot entangler. Three leads are coupled to three quantum dots in the Coulomb blockade regime. The central dot $D_{C}$ can accept 0,1 or 2 electrons provided from the source lead with rate $\alpha$; with 2 electrons, its ground state is the spin singlet. The tunnelling amplitudes $T_{0}$ describe the coherent tunnelling between $D_{C}$ and the secondary dots $D_{L}$ and $D_{R}$, which can only accept 0 or 1 electron. Each electron can finally tunnel out to the drain leads with a rate $\gamma$. (b) Single-particle energy level diagram. The dashed arrows represent the single-electron currents $I_{1}$ and $\tilde{I}_{1}$. Adapted from [15]. 
quantum dots $D_{L}$ and $D_{R}$, similarly to the resonance described in Sec. 2.1.1 . For this, we need the condition $\epsilon_{L}+\epsilon_{R}=2 \epsilon_{C}$, where $\epsilon_{L}$ and $\epsilon_{R}$ are the energy levels of the available state in $D_{L}$ and $D_{R}$, and $2 \epsilon_{C}$ is the total energy of the two electrons in $D_{C}$. On the other hand, the transport of a single electron from $D_{C}$ to $D_{L}$ or $D_{R}$ is suppressed by the energy mismatch $\epsilon_{C} \pm U \neq \epsilon_{L}, \epsilon_{R}$, where $\epsilon_{C} \pm U$ is the energy of the $2^{\text {nd }} / 1^{\text {st }}$ electron in $D_{C}$ [49].

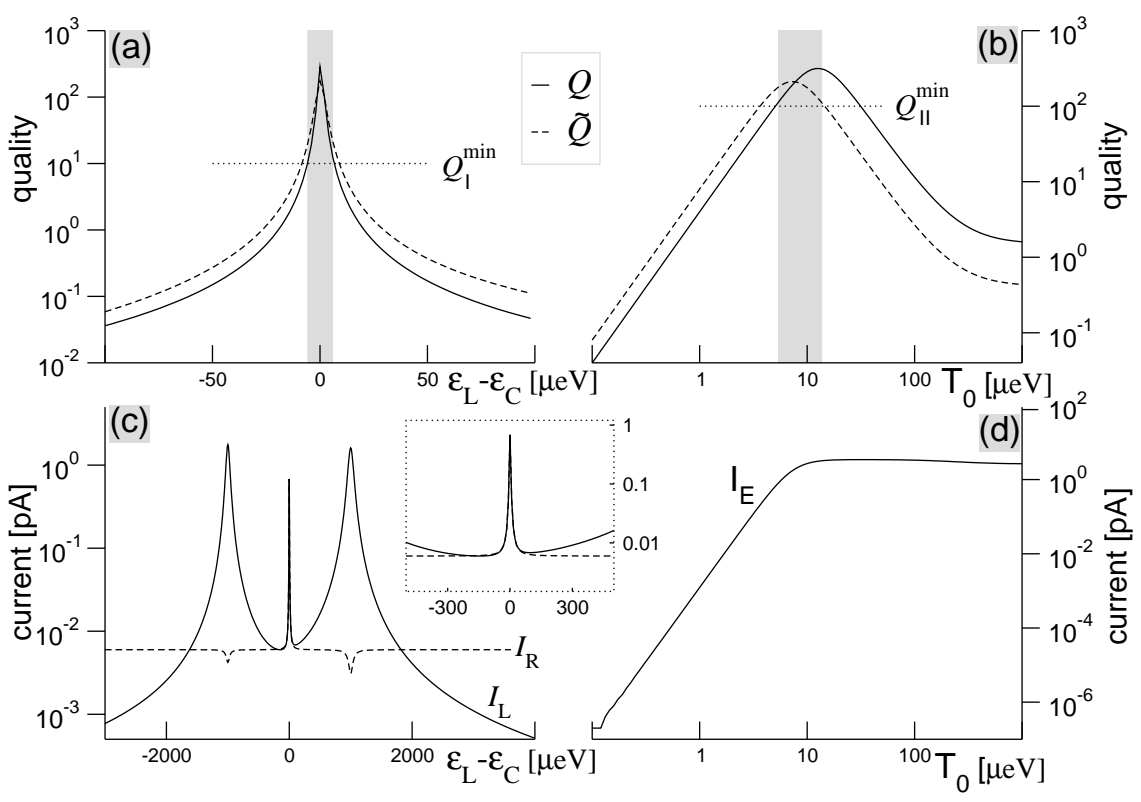

Figure 5. Quality and current of the entangler, with the parameters $\alpha=0.1, \gamma=1, T_{0}=10, U=1000$ in $\mu \mathrm{eV}$. (a) Quality $Q$ and $\widetilde{Q}$, around the resonance at $\epsilon_{L}-\epsilon_{C}=0$ where the entangled current dominates. In gray, the width of the resonance defined by $Q, \widetilde{Q}>Q_{\mathrm{I}}^{\mathrm{min}}=10$ is $\left|\epsilon_{L}-\epsilon_{C}\right|<6 \mu \mathrm{eV}$, as predicted by Eq.(6). (b) $Q$ and $\widetilde{Q}$ as a function of $T_{0}$ at resonance $\left(\epsilon_{L}=\epsilon_{C}\right)$. In gray, the region where the quality of the entangler is $Q, \widetilde{Q}>Q_{\mathrm{II}}^{\min }=100$ corresponding to Eq. (7). (c) Entangled and non-entangled current in the left $\left(I_{L}\right)$ and in the right $\left(I_{R}\right)$ drain leads. The inset shows the resonance in a larger scale. (d) Saturation of the entangled current $I_{E}$. Adapted from [15].

We describe the incoherent sequential tunneling between the leads and the dots in terms of a master equation [50] for the density matrix $\rho$ of the tripledot system (valid for $k_{\mathrm{B}} T>\gamma$ ). The stationary solution of the master equation is found with MAPLE, and is used to define stationary currents. Besides the entangled current $I_{E}$ coming from the joint transport of the electrons from $D_{C}$ to $D_{L}$ and $D_{R}$, the solitary escape of one electron of the singlet can create 
a non-entangled current $I_{1}$, as it could allow a new electron coming from the source lead to form a new spin-singlet with the remaining electron. Another non-entangled current $\tilde{I}_{1}$ can be present if only one electron is transported across the triple-dot system; see Fig. 4(b). The definition of entangler qualities $Q=I_{E} / I_{1}$ and $\tilde{Q}=I_{E} / \tilde{I}_{1}$ enables us to check the suppression of these non-entangled currents.

In Fig. 5 we present results in the case where $\epsilon_{R}=\epsilon_{C}$. This gives a twoelectron resonance at $\epsilon_{L}=\epsilon_{C}=\epsilon_{R}$, and create mobile entangled electrons with the same orbital energy, as required in the beam-splitter setup to allow entanglement detection [4], [8]. The exact analytical expressions are extremely lengthy, but we can get precise conditions for an efficient entangler regime by performing a Taylor expansion in terms of $\alpha, \gamma, T_{0}$ (defined in Fig. 4). Introducing the conditions $Q, \widetilde{Q}>Q_{\mathrm{I}}^{\min }$ away from resonance $\left(\epsilon_{L} \neq \epsilon_{C}\right)$ and $Q, \widetilde{Q}>Q_{\mathrm{II}}^{\min }$ at resonance $\left(\epsilon_{L}=\epsilon_{C}\right)$, we obtain the conditions [15]

$$
\begin{aligned}
& \left|\epsilon_{L}-\epsilon_{C}\right|<2 T_{0} / \sqrt{Q_{\mathrm{I}}^{\min }} \\
& \gamma \sqrt{Q_{\mathrm{II}}^{\min } / 8}<T_{0}<U \sqrt{4 \alpha / \gamma Q_{\mathrm{II}}^{\min }}
\end{aligned}
$$

We need a large $U$ for the energy suppression of the one-electron transport, and $\gamma \ll T_{0}$ because the joint transport is a higher-order process in $T_{0}$. The current saturates to $I_{E} \rightarrow e \alpha$ when $T_{0}^{4} \gg U^{2} \gamma \alpha / 32$ [see Fig. 5(d)] when the bottleneck process is the tunneling of the electrons from the source lead to the central dot. We see in (c) that equal currents in the left and right drain lead, $I_{L}=I_{R}$, are characteristic of the resonance $\epsilon_{L}=\epsilon_{C}$, which provides an experimental procedure to locate the efficient regime.

Taking realistic parameters for quantum dots $[25,51]$ such as $I_{E}=20 \mathrm{pA}$, $\alpha=0.1 \mu \mathrm{eV}$ and $U=1 \mathrm{meV}$, we obtain a maximum entangler quality $Q_{\mathrm{II}}^{\min }=100$ at resonance, and a finite width $\left|\epsilon_{L}-\epsilon_{C}\right| \simeq 6 \mu \mathrm{eV}$ where the quality is at least $Q_{\mathrm{I}}^{\min }=10$. Note that one must avoid resonances with excited levels which could favour the undesired non-entangled one-electron transport. For this, one can either tune the excited levels away by applying a magnetic field, or require a large energy levels spacing $\Delta \epsilon_{i} \simeq 2 U$, which can be found in vertical quantum dots or carbon nanotubes [25]. We can estimate the relevant timescales by simple arguments. The entangled pairs are delivered every $\tau_{\text {pairs }} \simeq 2 / \alpha \simeq 13 \mathrm{~ns}$. The average separation between two entangled electrons within one pair is given by the time-energy uncertainty relation: $\tau_{\text {delay }} \simeq 1 / U \simeq 0.6 \mathrm{ps}$, while their maximal separation is given by the variance of the exponential decay law of the escape into the leads: $\tau_{\max } \simeq 1 / \gamma \simeq 0.6 \mathrm{~ns}$. Note that $\tau_{\text {delay }}$ and $\tau_{\max }$ are both well below reported spin decoherence times (in bulk) of $100 \mathrm{~ns}$ [52]. 


\section{Spin-polarized electron sources}

Here we briefly mention some of the possibilities for spin-polarized electron sources possibly relevant as feeding Fermi-liquid reservoirs to our beamsplitter configuration. Even though we are concerned here with mesoscopic coherent transport, we emphasize that the electron sources themselves can be diffusive or ballistic.

Currently, there is a great deal of interest in the problem of spin injection in hybrid mesoscopic structures. At the simplest level we can say that the "Holy Grail" here is essentially the ability to spin inject and detect spin-polarized charge flow across interfaces. The possibility of controlling and manipulating the degree of spin polarization of the flow is highly desirable. This would enable novel spintronic devices with flexible/controllable functionalities.

Recently, many different experimental possibilities for spin injection/detection have been considered: (i) all-optical [53] and (ii) all-electrical [54], [55] spin injection and detection in semiconductors and metal devices, respectively, and (iii) electric injection with optical detection in hybrid (Mn-based) ferromagnetic/nonmagnetic and paramagnetic/non-magnetic semiconductor pin diodes [16]. For an account of the experimental efforts currently underway in the field of spinpolarized transport, we refer the reader to Ref. [13]. Below we focus on our proposals for spin filtering with a semimagnetic tunnel barrier [17] and a quantum dot [18]. These can, in principle, provide alternative schemes for spin injection into our beam splitter.

\subsection{QUANTUM SPIN FILTERING}

Ballistic Mn-based tunnel junctions [17] offer an interesting possibility for generating spin-polarized currents. Here the s-d interaction in the paramagnetic layer gives rise to a spin-dependent potential. An optimal design can yield high barriers for spin-up and vanishingly small barriers for spin-down electrons. Hence, a highly spin-selective tunnel barrier can be achieved in the presence of an external magnetic field. Note that here ballistic spin filtering - due to the blocking of one spin component of the electron flow - is the relevant mechanism for producing a spin-polarized current. Earlier calculations have shown that full spin polarizations are attainable in $\mathrm{ZnSe} / \mathrm{ZnMnSe}$ spin filters [17].

\subsection{QUANTUM DOTS AS SPIN FILTERS}

Spin polarized currents can also be generated by a quantum dot [18]. In the Coulomb blockade regime with Fermi-liquid leads, it can be operated as an efficient spin-filter [56] at the single electron level. A magnetic field lifts the spin degeneracy in the dot while its effect is negligible [57] in the leads. As a consequence, only one spin direction can pass through the quantum dot from 
the source to the drain. The transport of the opposite spin is suppressed by energy conservation and singlet-triplet splitting. This filtering effect can be enhanced by using materials with different $\mathrm{g}$-factors for the dot and the lead. To increase the current signal, one could also use an array of quantum dots, e.g. self-assembled dots.

\subsection{SPIN FILTERS FOR SPIN DETECTION AND BELL INEQUALITIES}

Besides being a source of spin-polarized currents, such spin filters (with or without spin-polarized sources [18],[58]) could be used to measure electron spin, as they convert spin information into charge: the transmitted charge current depends on the spin direction of the incoming electrons [26]. Such filters could probe the degree of polarization of the incoming leads. In addition, Bell inequalities measurements could be performed with such devices $[59,60]$.

\section{Scattering formalism: basics}

Current. In a multi-probe configuration with incoming and outgoing leads related via the scattering matrix $\mathbf{s}_{\gamma \beta}$, the current operator in lead $\gamma$ within the Landauer-Büttiker [61] approach is given by

$$
\begin{gathered}
\hat{I}_{\gamma}(t)=\frac{e}{h} \sum_{\alpha \beta} \int d \varepsilon d \varepsilon^{\prime} e^{i\left(\varepsilon-\varepsilon^{\prime}\right) t / \hbar} \mathbf{a}_{\alpha}^{\dagger}(\varepsilon) \mathbf{A}_{\alpha, \beta}\left(\gamma ; \varepsilon, \varepsilon^{\prime}\right) \mathbf{a}_{\beta}\left(\varepsilon^{\prime}\right), \\
\mathbf{A}_{\alpha \beta}\left(\gamma ; \varepsilon, \varepsilon^{\prime}\right)=\delta_{\gamma \alpha} \delta_{\gamma \beta} \mathbf{1}-\mathbf{s}_{\gamma \alpha}^{\dagger}(\varepsilon) \mathbf{s}_{\gamma \beta}\left(\varepsilon^{\prime}\right),
\end{gathered}
$$

where we have defined the two-component object $\mathbf{a}_{\alpha}^{\dagger}(\varepsilon)=\left(a_{\alpha, \uparrow}^{\dagger}(\varepsilon), a_{\alpha, \downarrow}^{\dagger}(\varepsilon)\right)$ with $a_{\alpha, \sigma}^{\dagger}(\varepsilon)$ denoting the usual fermionic creation operator for an electron with energy $\varepsilon$ and spin component $\sigma=\uparrow, \downarrow$ in lead $\alpha$. Here the spin components $\sigma$ are along a properly defined quantization axis (e.g., $x, y$ or $z$ ).

Noise. Let $\delta \hat{I}_{\gamma}(t)=\hat{I}_{\gamma}(t)-\langle I\rangle$ denote the current-fluctuation operator at time $t$ in lead $\gamma(\langle I\rangle$ : average current). We define noise between leads $\gamma$ and $\mu$ in a multi-terminal system by the average power spectral density of the symmetrized current-fluctuation autocorrelation function [62]

$$
S_{\gamma \mu}(\omega)=\frac{1}{2} \int\left\langle\delta \hat{I}_{\gamma}(t) \delta \hat{I}_{\mu}\left(t^{\prime}\right)+\delta \hat{I}_{\mu}\left(t^{\prime}\right) \delta \hat{I}_{\gamma}(t)\right\rangle e^{i \omega t} d t
$$

The angle brackets in Eq. (9) denote either an ensemble average or an expectation value between relevant pairwise electron states. We focus on noise at zero temperatures. In this regime, the current noise is solely due to the discreteness of the electron charge and is termed shot noise. 


\subsection{SCATTERING MATRIX}

Electron beam splitter. This device consists of four quasi one-dimensional leads (point contacts) electrostatically defined on top of a 2DEG [21], [22]. An extra "finger gate" in the central part of the device acts as a potential barrier for electrons traversing the system, i.e., a "beam splitter". That is, an impinging electron from, say, lead 1 has probability amplitudes $r$ to be reflected into lead 3 and $t$ to be transmitted into lead 4 .

Beam splitter $\mathbf{s}$ matrix. The transmission processes at the beam splitter can be suitably described in the language of the scattering theory: $s_{13}=s_{31}=r$ and $s_{14}=s_{41}=t$; similarly, $s_{23}=s_{32}=t$ and $s_{24}=s_{42}=r$, see Fig. 6 . We also neglect backscattering into the incoming leads, $s_{12}=s_{34}=s_{\alpha \alpha}=0$. Note that the beam splitter $\mathbf{s}$ matrix is spin independent; this no longer holds in the presence of a spin-orbit interaction. We also assume that the amplitudes $r$ and $t$ are energy independent. The unitarity of $\mathbf{s}$ implies $|r|^{2}+|t|^{2}=1$ and $\operatorname{Re}\left(r^{*} t\right)=0$. Below we use the above scattering matrix to evaluate noise.

\section{Noise of entangled electron pairs: earlier results}

Singlet and triplets. Let us assume that an entangler is now "coupled" to the beam-splitter device so as to inject entangled (and unentangled) electron pairs into the incoming leads, Fig. 6 . This will certainly require some challenging lithographic patterning and/or elaborate gating structures.

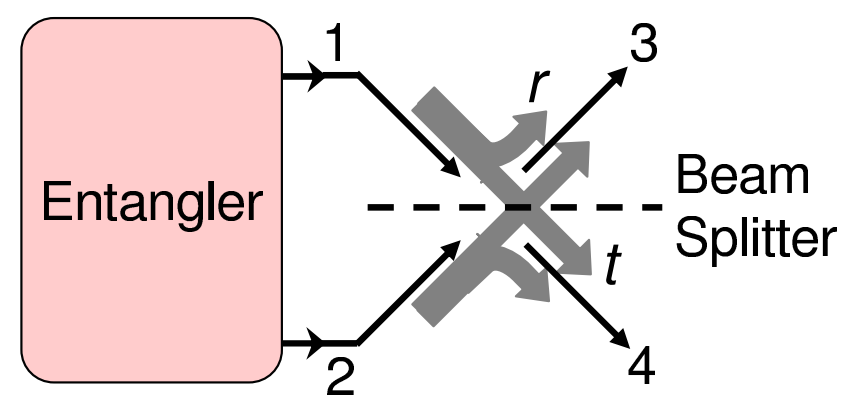

Figure 6. Electron entangler coupled to a beam splitter device. "Entangler" here represents one of the proposed setups of Sec. 2 or some other arrangement providing both triplet and singlet pairs via proper level tuning with gate electrodes. Adapted from Ref. [4].

Let us consider the following two-electron states

$$
|S\rangle=\frac{1}{\sqrt{2}}\left[a_{1 \uparrow}^{\dagger}\left(\varepsilon_{1}\right) a_{2 \downarrow}^{\dagger}\left(\varepsilon_{2}\right)-a_{1 \downarrow}^{\dagger}\left(\varepsilon_{1}\right) a_{2 \uparrow}^{\dagger}\left(\varepsilon_{2}\right)\right]|0\rangle,
$$




$$
|T e\rangle=\frac{1}{\sqrt{2}}\left[a_{1 \uparrow}^{\dagger}\left(\varepsilon_{1}\right) a_{2 \downarrow}^{\dagger}\left(\varepsilon_{2}\right)+a_{1 \downarrow}^{\dagger}\left(\varepsilon_{1}\right) a_{2 \uparrow}^{\dagger}\left(\varepsilon_{2}\right)\right]|0\rangle,
$$

and

$$
\left|T u_{\sigma}\right\rangle=a_{1 \sigma}^{\dagger}\left(\varepsilon_{1}\right) a_{2 \sigma}^{\dagger}\left(\varepsilon_{2}\right)|0\rangle, \quad \sigma=\uparrow, \downarrow .
$$

The above states correspond to the singlet $|S\rangle$, the entangled triplet $|T e\rangle$, and the unentangled triplets $\left|T u_{\sigma}\right\rangle$, respectively, injected electron pairs. Note that $|0\rangle$ denotes the "lead vacuum", i.e., an empty lead or a Fermi sea. Here we follow Ref. [4] and assume that the injected pairs have discrete energies $\varepsilon_{1,2}$. To determine the average current and shot noise for electron pairs we have to calculate the expectation value of the noise two-particle states in Eqs. (10)(12). In the limit of zero bias, zero temperature, and zero frequency, we find [4]

$$
S_{33}^{S / T e, u_{\sigma}}=\frac{2 e^{2}}{h \nu} T(1-T)\left(1 \pm \delta_{\varepsilon_{1}, \varepsilon_{2}}\right),
$$

for the shot noise in lead 3 for singlet (upper sign) and triplets (lower sign) with $T \equiv|t|^{2}$ (transmission coefficient). The corresponding currents in lead 3 are $I_{3}^{S, T e, u_{\sigma}}=I=\frac{e}{h \nu}$. Note the density of states factor $\nu$ in Eqs. (13) arising from the discrete spectrum used [63].

Bunching and antibunching. For $\varepsilon_{1}=\varepsilon_{2}$ the Fano factors corresponding to the shot noise in Eq. (13) are $F^{S}=S_{33}^{S} / e I=4 T(1-T)$, for the singlet and $F^{T e, u_{\sigma}}=0$, for all three triplets. Interestingly, the Fano factor for a singlet pair is enhanced by a factor of two as compared to the Fano factor $2 T(1-T)$ for a single uncorrelated electron beam [64] impinging on the beam splitter; the Fano factor for the triplets is suppressed with respect to this uncorrelated case. This enhancement of $F^{S}$ and suppression of $F^{T e, u_{\sigma}}$ is due to bunching and antibunching, respectively, of electrons in the outgoing leads. This result offers the possibility of distinguishing singlet from triplet states via noise measurements (triplets cannot be distinguished among themselves here; a further ingredient is needed for this, e.g., a local Rashba interaction in one of the incoming leads).

\section{Electron transport in the presence of a local Rashba s-o interaction}

The central idea here is to use the gate-controlled Rashba coupling to rotate the electron spins [24] traversing the Rashba-active region (lead 1 of the beam splitter), thus altering in a controllable way the resulting transport properties of the system. Below we first discuss the effects of the Rashba s-o interaction in one-dimensional systems; the incoming leads are essentially quasi onedimensional wires, i.e, "quantum point contacts". A local Rashba interaction can in principle be realized with an additional gating structure (top and back gates [65]). 
We focus on wires with one and two transverse channels [66]. This latter case allows us to study the effects of s-o induced interband coupling on both current and shot noise.

\subsection{RASHBA WIRES WITH UNCOUPLED TRANSVERSE CHANNELS}

\subsubsection{Hamiltonian, eigenenergies and eigenvectors}

The Rashba spin-orbit interaction is present in low-dimensional systems with structural inversion asymmetry. Roughly speaking, this interaction arises from the gradient of the confining potential ("triangular shape") at the interface between two different materials [67]. For a non-interacting one-dimensional wire with uncoupled transverse channels, the electron Hamiltonian in the presence of the Rashba coupling $\alpha$ reads [68]

$$
H_{n}=-\frac{\hbar^{2}}{2 m^{*}} \partial_{x}^{2}+\epsilon_{n}+i \alpha \sigma_{y} \partial_{x}
$$

In Eq. (14) $\partial_{x} \equiv \partial / \partial x, \sigma_{y}$ is the Pauli matrix, $m^{*}$ is the electron effective mass, and $\epsilon_{n}$ is the bottom of the $\mathrm{n}^{\text {th }}$-channel energy band in absence of s-o interaction. For an infinite-barrier transverse confinement of width $w, \epsilon_{n}=$ $n^{2} \pi^{2} \hbar^{2} /\left(2 m w^{2}\right)$.

The Hamiltonian in (14) yields the usual set of Rashba bands [69]

$$
\varepsilon_{s}^{n}=\hbar^{2}\left(k-s k_{R}\right)^{2} / 2 m^{*}+\epsilon_{n}-\epsilon_{R}, \quad s= \pm
$$

where $k_{R}=m^{*} \alpha / \hbar^{2}$ and $\epsilon_{R}=\hbar^{2} k_{R}^{2} / 2 m^{*}=m^{*} \alpha^{2} / 2 \hbar^{2}$ ("Rashba energy"). The corresponding wave functions are eigenvectors of $\sigma_{y}$ with the orbital part being a plane wave times the transverse-channel wave function. Figure 7 shows that the parabolic bands are shifted sideways due to the Rashba interaction. Note that these bands are still identified by a unique spin index $s= \pm$ which in our convention corresponds to the eigenspinors $|\mp\rangle \sim \mid \uparrow$ \rangle$\mp|\downarrow\rangle$ of $\sigma_{y}$.

\subsubsection{Boundary conditions and spin injection}

Here we assume a unity transmission across the interface [70] depicted in Fig. 7. For a spin-up electron with wave vector $k_{F}$ entering the Rashba region at $x=0$, we have the following boundary conditions for the wave function and its derivative $[69,71]$

$$
\left.|\uparrow\rangle e^{i k_{F} x}\right|_{x \rightarrow 0^{-}}=\frac{1}{\sqrt{2}}\left[|+\rangle e^{i k_{2} x}+|-\rangle e^{i k_{1} x}\right]_{x \rightarrow 0^{+}},
$$

and

$$
\left.|\uparrow\rangle v_{k_{F}} e^{i k_{F} x}\right|_{x \rightarrow 0^{-}}=\frac{1}{\sqrt{2}}\left[|+\rangle v_{k_{2}}^{R} e^{i k_{2} x}+|-\rangle v_{k_{1}}^{R} e^{i k_{1} x}\right]_{x \rightarrow 0^{+}},
$$




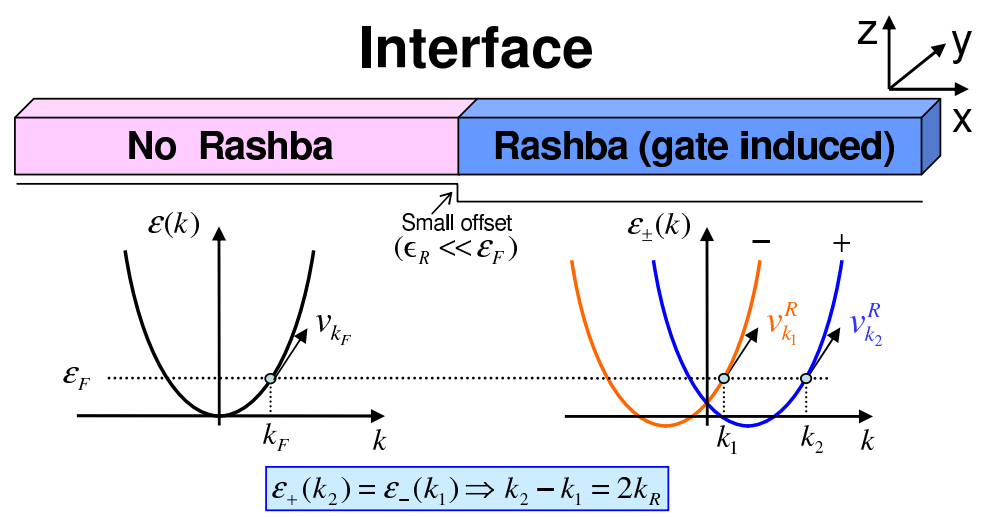

Figure 7. Schematic of a portion of a gate-induced no-Rashba/Rashba "interface" and its corresponding band structure. Note the small band offset arising solely from the mismatch $\epsilon_{R}$.

with the Fermi and Rashba group velocities defined by $v_{k_{F}}=\hbar k_{F} / m^{*}$, $v_{k_{1}}^{R}=\frac{\hbar}{m^{*}}\left(k_{1}+k_{R}\right)$, and $v_{k_{2}}^{R}=\frac{\hbar}{m^{*}}\left(k_{2}-k_{R}\right)$. The wave vectors $k_{1}$ and $k_{2}$ are defined by the "horizontal" intersections with the Rashba bands $\varepsilon_{-}\left(k_{1}\right)=$ $\varepsilon_{+}\left(k_{2}\right)$, see Fig. 7. This results in the condition $k_{2}-k_{1}=2 k_{R}$ which implies that the Rashba group velocities are the same at these points: $v_{k_{1}}^{R}=v_{k_{2}}^{R}$. Equation (17) is satisfied provided that [69]

$$
v_{k_{F}}=\frac{1}{2}\left(v_{k_{1}}^{R}+v_{k_{2}}^{R}\right)=\frac{\hbar}{m^{*}} \sqrt{\frac{2 m^{*}}{\hbar^{2}}\left(\varepsilon_{F}+\epsilon_{R}\right)},
$$

where the last equality follows from conservation of energy, $\varepsilon_{-}\left(k_{1}\right)=\varepsilon_{+}\left(k_{2}\right)=$ $\varepsilon_{F}$. Note that the group velocity of the incoming spin-up electron is completely "transferred" to the Rashba states at the interface.

Spin-rotated state at $x=L$. For an incoming spin-up electron, we have at the exit of the Rashba region the spin-rotated state

$$
\psi_{\uparrow, L}=\frac{1}{\sqrt{2}}\left[|+\rangle e^{i k_{2} L}+|-\rangle e^{i k_{1} L}\right]
$$

which is consistent with the boundary conditions (16) and (17). After some straightforward manipulations (and using $k_{2}-k_{1}=2 k_{R}$ ), we find

$$
\psi_{\uparrow, L}=\left(\begin{array}{c}
\cos \theta_{R} / 2 \\
\sin \theta_{R} / 2
\end{array}\right) e^{i\left(k_{1}+k_{R}\right) L},
$$

with the usual Rashba angle $\theta_{R}=2 m^{*} \alpha L / \hbar^{2}[24,72]$. A similar expression holds for an incoming spin-down electron. Note that the boundary conditions at $x=L$ are trivially satisfied since we assume unity transmission. The 
overall phase of the spinor in Eq. (20) is irrelevant for our purposes; we shall drop it from now on.

\subsubsection{Rashba spin rotator}

From the results of the previous section we can now define a unitary operator which describes the action of the Rashba-active region on any incoming spinor

$$
\mathbf{U}_{\mathbf{R}}=\left(\begin{array}{cc}
\cos \theta_{R} / 2 & -\sin \theta_{R} / 2 \\
\sin \theta_{R} / 2 & \cos \theta_{R} / 2
\end{array}\right) .
$$

Note that all uncoupled transverse channels are described by the same unitary operator $\mathbf{U}_{\mathbf{R}}$. The above unitary operator allows us to incorporate the s-o induced precession effect straightforwardly into the scattering formalism (Sec. 7).

\subsection{RASHBA WIRE WITH TWO COUPLED TRANSVERSE CHANNELS}

The Rashba s-o interaction also induces a coupling between the bands described in the previous section. Here we extend our analysis to the case of two weakly coupled Rashba bands.

\subsubsection{Exact and approximate energy bands}

Projecting the two-dimensional Rashba Hamiltonian [68] onto the basis of the two lowest uncoupled Rashba states, we obtain the quasi one-dimensional Hamiltonian [72]

$$
H=\left[\begin{array}{cccc}
\varepsilon_{+}^{a}(k) & 0 & 0 & -\alpha d \\
0 & \varepsilon_{-}^{a}(k) & \alpha d & 0 \\
0 & \alpha d & \varepsilon_{+}^{b}(k) & 0 \\
-\alpha d & 0 & 0 & \varepsilon_{-}^{b}(k)
\end{array}\right]
$$

where the interband coupling matrix element is $d \equiv\left\langle\phi_{a}(y)|\partial / \partial y| \phi_{b}(y)\right\rangle$ and $\phi_{n}(y)$ is the transverse channel wave function. Here we label the uncoupled Rashba states by $n=a, b$ [73]. The Hamiltonian above gives rise to two sets of parabolic Rashba bands for zero interband coupling $d=0$. These bands are sketched in Fig. 8 (thin lines). Note that the uncoupled Rashba bands cross. For positive $k$ vectors the crossing is at $k_{c}=\left(\epsilon_{b}-\epsilon_{a}\right) / 2 \alpha$. For nonzero interband coupling $d \neq 0$ the bands anti-cross near $k_{c}$ (see thick lines); this follows from a straightforward diagonalization of the $4 \mathrm{x} 4$ matrix in Eq. (22). We are interested here in the weak interband coupling limit. In addition, we consider electron energies near the crossing; away from the crossing the bands are essentially uncoupled and the problem reduces to that of the previous section. In what follows, we adopt a perturbative description for the energy bands near $k_{c}$ which allows us to obtain analytical results. 


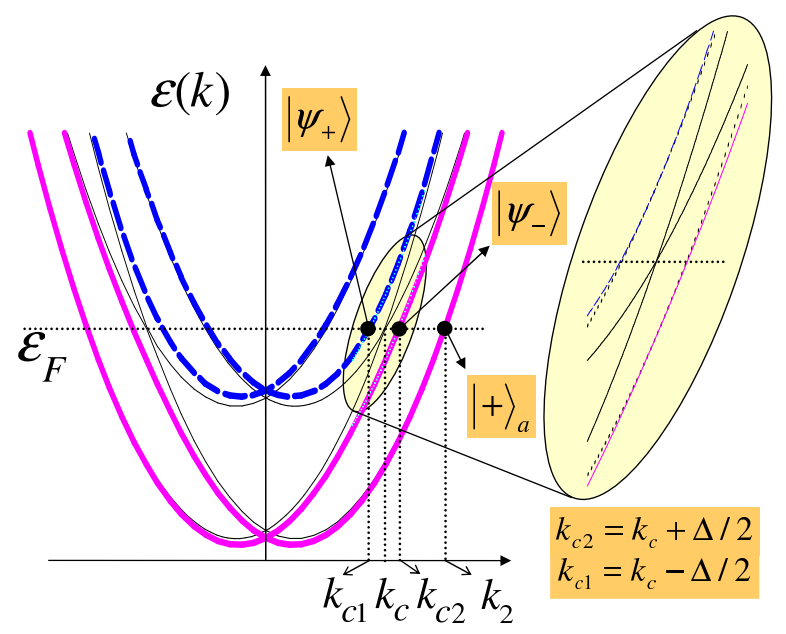

Figure 8. Band structure for a wire with two sets of Rashba bands. Both the uncoupled (thin lines) and the interband-coupled (thick solid and dashed lines) are shown. The uncoupled Rashba bands cross at $k_{c}$. Spin-orbit induced interband coupling gives rise to anti crossing of the bands near $k_{c}$. Inset: blowup of the region near the crossing. The nearly-free electron bands [perturbative approach, Eq. (24)] describe quite well the exact dispersions near the crossing (cf. dotted and solid + dashed lines in the inset). The solid circles ("intersections") indicate the relevant $k$ points for spin injection [Eq. (27)]; their corresponding zeroth-order eigenvectors [Eq. (25)] are also indicated.

"Nearly-free electron bands". In analogy to the usual nearly-free electron approach in solids [74], we restrict the diagonalization of Eq. (22) to the $2 \times 2$ central block which corresponds to the degenerate Rashba states crossing at $k_{c}$

$$
\tilde{H}=\left[\begin{array}{cc}
\varepsilon_{-}^{a}(k) & \alpha d \\
\alpha d & \varepsilon_{+}^{b}(k)
\end{array}\right]
$$

To lowest order we find

$$
\varepsilon_{ \pm}^{\text {approx }}(k)=\frac{\hbar^{2} k^{2}}{2 m}+\frac{1}{2} \epsilon_{b}+\frac{1}{2} \epsilon_{a} \pm \alpha d
$$

The corresponding eigenvectors are the usual linear combination of the zeroth order degenerate states at the crossing

$$
\left|\psi_{ \pm}\right\rangle=\frac{1}{\sqrt{2}}\left[|-\rangle_{a} \pm|+\rangle_{b}\right]
$$

where the ket sub-indices denote the respective (uncoupled) Rashba channel [for simplicity, we omit the orbital part of the wave functions in (25)]. 


\subsubsection{Boundary conditions and spin injection near the crossing}

Here we extend the analysis in Sec. 6.1.2 to the case of two interband-coupled bands. We first determine the $k$ points corresponding to the "horizontal intersections" near the crossing at $k_{c}$, i.e., $k_{c 1}$ and $k_{c 2}$, see Fig. 8 . We need these points since incoming spin-up electrons will be primarily injected into those states (and also into $k_{2}$, conservation of energy). By defining $k_{c 1}=k_{c}-\Delta / 2$ and $k_{c 2}=k_{c}+\Delta / 2$ and then imposing $\varepsilon_{+}^{\text {approx }}\left(k_{c 1}\right)=\varepsilon_{-}^{\text {approx }}\left(k_{c 2}\right)$ (assumed $\sim \varepsilon_{F}$ ) we find,

$$
\Delta=\frac{2 m \alpha d}{\hbar^{2} k_{c}}=2 \frac{k_{R}}{k_{c}} d .
$$

For a spin-up electron in the lowest wire state in the "no-Rashba" region (channel $a$ ), we can again write at $x=0$ [70]

$$
\begin{aligned}
& \left.|\uparrow\rangle e^{i k x}\right|_{x \rightarrow 0^{-}}= \\
& \quad \frac{1}{\sqrt{2}}\left\{\frac{1}{\sqrt{2}}\left[\left|\psi_{+}\right\rangle e^{i k_{c 1} x}+\left|\psi_{-}\right\rangle e^{i k_{c 2} x}\right]+|+\rangle_{a} e^{i k_{2} x}\right\}_{x \rightarrow 0^{+}},
\end{aligned}
$$

in analogy to Eq. (16). Note that we only need to include three intersection points in the above "expansion" since the incoming spin-up electron is in channel $a$. Equation (27) satisfies the continuity of the wave function. The boundary condition for the derivative of the wave function is also satisfied provide that $\Delta / 4 \ll k_{F}$. This condition is readily fulfilled for realistic parameters (Sec. 8.3). Hence, fully spin-polarized injection into the Rashba region is still possible in the presence of a weak interband coupling. Here we are considering a fully spin-polarized injector so that the intrinsic limitation due to the "conductivity mismatch" [75] is not a factor.

Generalized spin-rotated state at $x=L$. Here again we can easily determine the form of the state at the exit of the Rashba region. For an incoming spin-up electron in the lowest band of the wire, we find

$$
\Psi_{\uparrow, L}=\frac{1}{2} e^{i\left(k_{c}+k_{R}\right) L}\left(\begin{array}{c}
\cos \left(\theta_{d} / 2\right) e^{-i \theta_{R} / 2}+e^{i \theta_{R} / 2} \\
-i \cos \left(\theta_{d} / 2\right) e^{-i \theta_{R} / 2}+i e^{i \theta_{R} / 2} \\
-i \sin \left(\theta_{d} / 2\right) e^{-i \theta_{R} / 2} \\
\sin \left(\theta_{d} / 2\right) e^{-i \theta_{R} / 2}
\end{array}\right) .
$$

A similar state holds for a spin-down incoming electron. The state (28) satisfies the boundary conditions at $x=L$ (again, provided that $\Delta \ll 4 k_{F}$. Equation (28) essentially tells us that a weak s-o interband coupling gives rise to an additional spin rotation (besides $\theta_{R}$ ) described by the mixing angle $\theta_{d}=\theta_{R} d / k_{c}$. This extra modulation enhances spin control in a Datta-Das spin-transistor geometry. In Ref. [72] we show that the spin-resolved current in this case is

$$
I_{\uparrow, \downarrow}=\frac{e}{h} e V\left[1 \pm \cos \left(\theta_{d} / 2\right) \cos \theta_{R}\right],
$$


where $V$ is the source-drain bias.

\section{Novel Beam-splitter geometry with a local Rashba interaction}

Figure 1 shows an schematic of our proposed beam-splitter geometry with a local Rashba-active region of length $L$ in lead 1 . Below we discuss its scattering matrix in the absence of interband coupling. In this case, each set of Rashba bands can be treated independently.

Combined $\mathbf{s}$ matrices. An electron entering the system through port 1, first undergoes a unitary Rashba rotation $\mathbf{U}_{\mathbf{R}}$ in lead 1 then reaches the beam splitter which either reflects the electron into lead 3 or transmits it into lead 4. This happens for electrons injected into either the first or the second set of uncoupled Rashba bands. Since the Rashba spin rotation is unitary, we can combine the relevant matrix elements of the beam-splitter $\mathbf{s}$ matrix, connecting leads 1 and $3\left(s_{14}=s_{41}\right)$ and 1 and $4\left(s_{14}=s_{41}\right)$, with the Rashba rotation matrix $\mathbf{U}_{\mathbf{R}}$ thus obtaining effective spin-dependent $2 \times 2$ matrices of the form $\mathbf{s}_{\mathbf{1 3}}^{\mathbf{R}}=\mathbf{s}_{\mathbf{3 1}}^{\mathbf{R}}=s_{13} \mathbf{U}_{\mathbf{R}}$ A similar definition holds for $\mathbf{s}_{\mathbf{1 4}}^{\mathbf{R}}=s_{41} \mathbf{U}_{\mathbf{R}}=\mathbf{s}_{\mathbf{4 1}}^{\mathbf{R}}$. Note also that $\mathbf{s}_{23}=\mathbf{s}_{32}=t \mathbf{1}$ and $\mathbf{s}_{\mathbf{2 4}}=\mathbf{s}_{\mathbf{4}}=r \mathbf{1}$ since no Rashba coupling is present in lead 2. All the other matrix elements are zero. Hence the new effective beam-splitter s matrix which incorporates the effect of the Rashba interaction in lead 1 reads

$$
\mathrm{s}=\left(\begin{array}{cccc}
0 & 0 & \mathrm{~s}_{13}^{\mathrm{R}} & \mathrm{s}_{14}^{\mathrm{R}} \\
0 & 0 & \mathrm{~s}_{23} & \mathrm{~s}_{24} \\
\mathrm{~s}_{31}^{\mathrm{R}} & \mathrm{s}_{32} & 0 & 0 \\
\mathrm{~s}_{41}^{\mathrm{R}} & \mathrm{s}_{42} & 0 & 0
\end{array}\right)
$$

Note that incorporating the s-o effects directly into the beam-splitter scattering matrix makes it spin dependent. The Rashba interaction does not introduce any noise in lead 1 . This is so because the electron transmission coefficient through lead 1 is essentially unity [70]; a quantum point contact is noiseless for unity transmission.

Coupled Rashba bands. The interband-coupled case can, in principle, be treated similarly. However, we follow a different simpler route to determine the shot noise in this case. We discuss this in more detail in Sec. 8.1.2.

\section{Noise of entangled and spin-polarized electrons in the presence of a local Rashba spin-orbit interaction}

Starting from the noise definition in (Eq. 9), we briefly outline here the derivation of noise expressions for pairwise electron states (entangled and unentangled) and spin-polarized electrons (Secs. 2 and 3). For each of these two cases, we present results with and without s-o induced interband coupling. 


\subsection{SHOT NOISE FOR SINGLET AND TRIPLETS}

\subsubsection{Uncoupled Rashba bands: single modulation $\theta_{R}$}

To determine noise, we calculate the expectation value of the noise operator (Eq. 9) between pairwise electron states. We have derived shot noise expressions for both singlet and triplet states for a generic spin-dependent $\mathbf{s}$ matrix. Our results quite generally show that unentangled triplets and the entangled triplet display distinctive shot noise for spin-dependent scattering matrices. Below we present shot noise formulas for the specific case of interest here; namely, the beam-splitter scattering matrix in the presence of a local Rashba term [Eq. (30)]. In this case, for singlet and triplets defined along different quantization axes ( $\hat{x}$ and $\hat{z}$ are equivalent directions perpendicular to the Rashba rotation axis $-\hat{y}$ ), we find

$$
\begin{gathered}
S_{33}^{S}\left(\theta_{R}\right)=\frac{2 e^{2}}{h \nu} T(1-T)\left[1+\cos \left(\theta_{R}\right) \delta_{\varepsilon_{1}, \varepsilon_{2}}\right], \\
S_{33}^{T e_{y}}\left(\theta_{R}\right)=\frac{2 e^{2}}{h \nu} T(1-T)\left[1-\cos \left(\theta_{R}\right) \delta_{\varepsilon_{1}, \varepsilon_{2}}\right], \\
S_{33}^{T e_{z}}\left(\theta_{R}\right)=S_{s-o}^{T_{u_{y}}}\left(\theta_{R}\right)=\frac{2 e^{2}}{h \nu} T(1-T)\left(1-\delta_{\varepsilon_{1}, \varepsilon_{2}}\right),
\end{gathered}
$$

and

$$
S_{33}^{T u_{\uparrow}}\left(\theta_{R}\right)=S_{33}^{T u_{\downarrow}}\left(\theta_{R}\right)=\frac{2 e^{2}}{h \nu} T(1-T)\left[1-\cos ^{2}\left(\theta_{R} / 2\right) \delta_{\varepsilon_{1}, \varepsilon_{2}}\right] .
$$

Equations (32)-(34) clearly show that entangled and unentangled triplets present distinct noise as a functions of the Rashba phase. Note that for $\theta_{R}=0$, we regain the formulas in Sec. 5.

Figure 9 shows the "reduced" Fano factor $f=F / 2 T(1-T), F=S_{33} / e I$ (here $I=e / h \nu$ ), as a function of the Rashba angle $\theta_{R}$ for the noise expressions (31)-(34). We clearly see that singlet and triplet pairs exhibit distinct shot noise in the presence of the s-o interaction. The singlet $S$ and entangled (along the Rashba rotation axis $\hat{y}$ ) triplet $T e_{y}$ pairs acquire an oscillating phase in lead 1 thus originating intermediate degrees of bunching/antibunching (solid and dotted lines, respectively). Triplet states (entangled and unentangled) display distinctive noise as a function of the Rashba phase, e.g., $T e_{y}$ is noisy and $T u_{y}$ is noiseless. Hence entangled and unentangled triplets can also be distinguished via noise measurements. Note that for $\theta_{R}=0$ all three triplets exhibit identically zero noise [see Eq.(13)].

\subsubsection{Interband-coupled Rashba bands: additional modulation $\theta_{d}$}

Here we determine noise for injected pairs with energies near the crossing $\varepsilon\left(k_{c}\right)$ using an alternate scheme. We calculate the relevant expectation values 


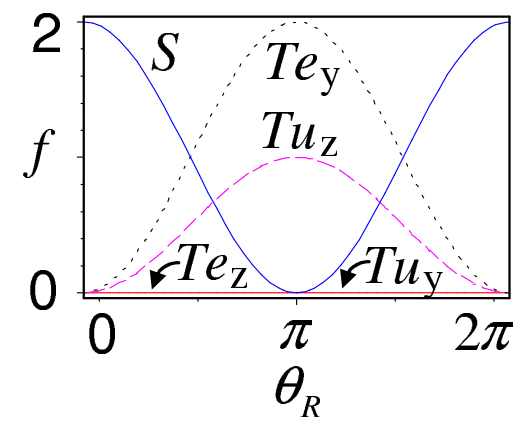

Figure 9. "Reduced" Fano factor $f$ as a function of the Rashba phase for singlet and triplets along different quantization axes. Note that singlet and entangled triplet states show continuous bunching and antibunching behaviors as $\theta_{R}$ is increased. Unentangled triplets display distinctive noise for a given polarization and for different polarizations. Adapted from Ref. [8].

of the noise by using pairwise states defined from the generalized spin-rotated state in Eq. (28) and its spin-down counterpart. Since these states already incorporate all the relevant effects (Rashba rotation and interband mixing), we can calculate noise by using the "bare" beam splitter matrix elements, generalized to account for two channels. The beam-splitter does not mix transverse channels; hence this extension is trivial, i.e., block diagonal in the channel indices. This approach was first developed in Ref. [7].

Rashba-evolved pairwise electron states. The portion of an electron-pair wave function "propagating" in lead 1 undergoes the effects of the Rashba interaction: ordinary precession $\theta_{R}$ and additional rotation $\theta_{d}$. Using Eq. (28) (and its spin-down counterpart) we find the following states

$$
\begin{aligned}
\left|S / T e_{z}\right\rangle_{L}= & \frac{1}{2}\left[\cos \left(\theta_{d} / 2\right) e^{-i \theta_{R} / 2}+e^{i \theta_{R} / 2}\right] \frac{|\uparrow \downarrow\rangle_{a a} \mp|\downarrow \uparrow\rangle_{a a}}{\sqrt{2}}+ \\
& \frac{1}{2}\left[-i \cos \left(\theta_{d} / 2\right) e^{-i \theta_{R} / 2}+i e^{i \theta_{R} / 2}\right] \frac{|\downarrow \downarrow\rangle_{a a} \pm|\uparrow \uparrow\rangle_{a a}}{\sqrt{2}}+ \\
& \frac{1}{2}\left[-i \sin \left(\theta_{d} / 2\right) e^{-i \theta_{R} / 2}\right] \frac{|\uparrow \downarrow\rangle_{b a} \pm|\downarrow \uparrow\rangle_{b a}}{\sqrt{2}}+ \\
& \frac{1}{2}\left[\sin \left(\theta_{d} / 2\right) e^{-i \theta_{R} / 2}\right] \frac{|\downarrow \downarrow\rangle_{b a} \mp|\uparrow \uparrow\rangle_{b a}}{\sqrt{2}} .
\end{aligned}
$$


The notation $\left|T e_{z}\right\rangle_{L}$ and $|S\rangle_{L}$ emphasizes the type of injected pairs (singlets or triplets at $x=0$ ) propagating through the length $L$ of the Rashba-active region in lead 1. Similar expressions hold for $\left|T u_{\uparrow, \downarrow}\right\rangle_{L}$. In addition, we use the shorthand notation $|\downarrow \uparrow\rangle_{b a} \equiv\left|\downarrow_{1 b} \uparrow_{2 a}\right\rangle$, denoting a pair with one electron in channel $b$ of lead 1 and another in channel $a$ of lead 2. Here we consider incoming pairs with $\hat{z}$ polarizations only. Despite the seemingly complex structure of the above pairwise states, they follow quite straightforwardly from the general state $\Psi_{\uparrow, L}$ in (28) (and its counterpart $\Psi_{\downarrow, L}$ ). For instance, the unentangled triplet $\left|T u_{\uparrow}\right\rangle_{L}$ is obtained from the tensor product between $\Psi_{\uparrow, L}$ [which describes as electron crossing lead 1 (initially spin up and in channel $a$ )] and a spin-up state in channel $a$ of lead 2: $\left|T u_{\uparrow}\right\rangle_{L}=\left|\Psi_{\uparrow, L}\right\rangle \otimes \mid \uparrow$ \rangle$_{2 a}$.

Noise. We can now use the above states to determine shot noise at the zero frequency, zero temperature, and zero applied bias. Using the shot-noise results of Sec. 5 (trivially generalized for two channels), we find for the noise in lead 3

$$
\begin{aligned}
S_{33}^{T u_{\uparrow}}\left(\theta_{R}, \theta_{d}\right)= & S_{33}^{T u_{\downarrow}}\left(\theta_{R}, \theta_{d}\right)=\frac{2 e^{2}}{h \nu} T(1-T) \times \\
& {\left[1-\frac{1}{2}\left(1+\cos \frac{\theta_{d}}{2} \cos \theta_{R}-\frac{1}{2} \sin ^{2} \frac{\theta_{d}}{2}\right) \delta_{\varepsilon_{1}, \varepsilon_{2}}\right], } \\
S_{33}^{T e_{z}}\left(\theta_{R}, \theta_{d}\right)= & \frac{2 e^{2}}{h \nu} T(1-T)\left[1-\frac{1}{2}\left(\cos ^{2} \frac{\theta_{d}}{2}+1\right) \delta_{\varepsilon_{1}, \varepsilon_{2}}\right],
\end{aligned}
$$

and

$$
S_{33}^{S}\left(\theta_{R}, \theta_{d}\right)=\frac{2 e^{2}}{h \nu} T(1-T)\left[1+\left(\cos \frac{\theta_{d}}{2} \cos \theta_{R}\right) \delta_{\varepsilon_{1}, \varepsilon_{2}}\right] .
$$

Equations (36)-(38) describe shot noise only for injected pairs with energies near the crossing, say, within $\alpha d$ of $\varepsilon\left(k_{c}\right)$. Away from the crossing or for $d=0$, the above expressions reduce to those of Sec. 8.1.1. We can also define "reduced" Fano factors as before; the interband mixing angle $\theta_{d}$ further modulates the Fano factors. For conciseness, we present the angular dependence of the Fano factors in the next section.

\subsection{SHOT NOISE FOR SPIN-POLARIZED ELECTRONS}

We have derived a general shot noise formula for the case of spin-polarized sources by performing the ensemble average in Eq. (9) over appropriate thermal reservoirs. The resulting expression corresponds to the standard LandauerBüttiker formula for noise with spin-dependent s matrices. Below we present results for the specific beam-splitter s matrix in (30). 
8.2.1. Uncoupled-band case: single modulation $\theta_{R}$

For incoming leads with a degree of spin polarization $p$ and for the scattering matrix (30), we find at zero temperatures

$$
S_{33}^{p}\left(\theta_{R}\right)=2 e I T(1-T) p \sin ^{2} \frac{\theta_{R}}{2},
$$

where $I=2 e^{2} V /[h(1+p)]$ is the average current in lead 3. The "reduced" Fano factor corresponding to Eq. (39) is $f_{p}=p \sin ^{2}\left(\theta_{R} / 2\right)$. Figure 10 shows $f_{p}$ as a function of the Rashba angle $\theta_{R}$. For spin polarized injection along the Rashba rotation axis $(-\hat{y})$ no noise results in lead 3 . This is a consequence of the Pauli exclusion principle in the leads. Spin-polarized currents with polarization perpendicular to the Rashba axis exhibit sizable oscillations as a function of $\theta_{R}$. Full shot noise is obtained for $\theta_{R}=\pi$ since the spin polarization of the incoming flow is completely reversed within lead 1 .

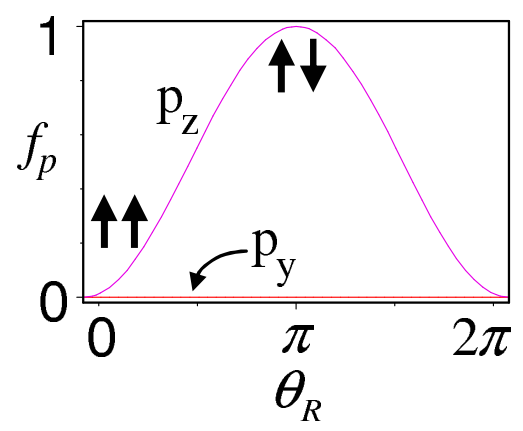

Figure 10. "Reduced" Fano factor for fully spin-polarized $(p=1)$ incoming beams in leads 1 and 2 as a function of the Rashba phase. Polarizations along two distinct quantization axes are shown $\left(p_{z}\right.$ and $\left.p_{z}\right)$. For spin injection along the Rashba rotation axis $(-\hat{y})$, no precession occurs in lead 1 and shot noise is identically zero (Pauli principle). Spin-polarized carriers injected along $\hat{z}$ undergo precession and hence exhibit shot noise. Adapted from Ref. [8].

Probing/detecting spin-polarized currents. Since unpolarized incoming beams in lead 1 and 2 yield zero shot noise in lead 3, the results shown in Fig. 10 provide us with an interesting way to detect spin-polarized currents via their noise. In addition, noise measurements should also allow one to probe the direction of the spin-polarization of the injected current. 
Measuring the s-o coupling. We can express the s-o coupling constant in terms of the reduced Fano factor. For a fully spin-polarized beam $(p=1)$, we have

$$
\alpha=\frac{\hbar^{2}}{m^{*} L} \arcsin \sqrt{f_{p}}
$$

Equation (40) provides a direct means of extracting the Rashba s-o coupling $\alpha$ via shot noise measurements. We can also obtain a similar expression for $\alpha$ from the unentangled triplet noise formula (34).

\subsubsection{Interband-coupled case: extra modulation $\theta_{d}$}

The calculation in the previous section can be extended to the interbandcoupled case for electrons impinging near the anti crossing of the bands $\left[\sim \varepsilon\left(k_{c}\right)\right]$. Here we present a simple "back-of-the-envelope" derivation of the the shot noise for the fully spin-polarized current case $(p=1)$ from that of the spin-up unentangled triplet Eq. (36). Here we imagine that the spectrum of the triplet $T u_{\uparrow}$ forms now a continuum and integrate its noise expression (after making $\varepsilon_{1}=\varepsilon_{2}$ ) over some energy range to obtain the noise of a spinpolarized current. Assuming $T$ constant in the range $\left(\varepsilon_{F}, \varepsilon_{F}+e V\right)$, we find to linear order in $\mathrm{eV}$

$$
S_{33}^{\uparrow}\left(\theta_{R}, \theta_{d}\right)=e I T(1-T)\left(1-\cos \frac{\theta_{d}}{2} \cos \theta_{R}+\frac{1}{2} \sin ^{2} \frac{\theta_{d}}{2}\right) .
$$

Figures 11(a) and 11(b) illustrate the angular dependencies of the reduced Fano factors for both the spin-polarized case Eq. (41) and that of the singlet Eq. (38). Note that the further modulation $\theta_{d}$ due to interband mixing can drastically change the noise for both spin-polarized and entangled electrons. For the singlet pairs, for instance, it can completely reverse the bunching/antibunching features. Hence further control is gained via $\theta_{d}$ which can, in principle, be tuned independently of $\theta_{R}$ (see Sec. 8.3).
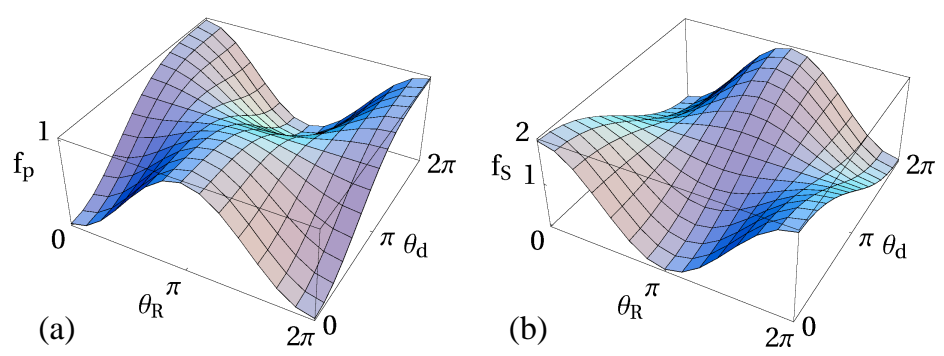

Figure 11. Reduced Fano factors $f=f_{p}$ (a) and $f_{S}$ (b), for fully spin-polarized ( $p=1, \hat{z}$ direction) incoming electrons and for singlet pairs, respectively, as a function of the Rashba angle $\theta_{R}$ and the interband mixing angle $\theta_{d}$. The additional phase $\theta_{d}$ can significantly alter the noise characteristics. 


\subsection{REALISTIC PARAMETERS: ESTIMATES FOR $\theta_{R}$ AND $\theta_{d}$.}

We conclude this section by presenting some estimates for the relevant spinrotation angles $\theta_{R}$ and $\theta_{d}$ for realistic system parameters. Let us assume, for the sake of concreteness, an infinite confining potential of width $w$. In this case, the transverse wire modes in absence of the Rashba interaction are quantized with energies $\epsilon_{n}=\hbar^{2} \pi^{2} n^{2} /\left(2 m^{*} w^{2}\right)$. Let us now set $\epsilon_{b}-$ $\epsilon_{a}=3 \hbar^{2} \pi^{2} /\left(2 m^{*} w^{2}\right)=16 \epsilon_{R}$ which is a "reasonable guess". Since $\epsilon_{R}=$ $m^{*} \alpha^{2} / 2 \hbar^{2}$, we find $\alpha=(\sqrt{3} \pi / 4) \hbar^{2} / m^{*} w^{2}=3.45 \times 10^{-11} \mathrm{eVm}$ [65] (which yields $\epsilon_{R} \sim 0.39 \mathrm{meV}$ ) for $m^{*}=0.05 m_{0}$ and $w=60 \mathrm{~nm}$. For the above choice of parameters, the energy at the crossing is $\epsilon_{-}^{a}\left(k_{c}\right)=\epsilon_{+}^{b}\left(k_{c}\right)=$ $\epsilon\left(k_{c}\right)=24 \epsilon_{R} \sim 9.36 \mathrm{meV}$. Electrons with energies around this value are affected by the s-o interband coupling, i.e., they undergo the additional spin rotation $\theta_{d}$. The relevant wave vector at the crossing is $k_{c}=8 \epsilon_{R} / \alpha$. Assuming the $L=69 \mathrm{~nm}$ for the length of the Rashba channel, we find $\theta_{R}=\pi$ and $\theta_{d}=\theta_{R} d / k_{c} \sim \pi / 2$ since $d / k_{c}=2 /\left(3 k_{R} w\right)$ and $k_{R} w=\sqrt{3} \pi / 4 \sim 4 / 3$ for $\epsilon_{b}-\epsilon_{a}=16 \epsilon_{R}$ which implies $d / k_{c} \sim 0.5$. The preceding estimates are conservative. We should point out that both $\theta_{R}$ and $\theta_{d}$ can, in principle, be varied independently via side gates. It should also be possible to "over rotate" $\theta_{R}$ (say, by using a larger $L$ ) and hence increase $\theta_{d}$. As a final point we note that $\Delta / 4 k_{F} \sim 0.05 \ll 1\left[k_{F}\right.$ is obtained by making $\left.\varepsilon_{F}=\hbar^{2} k_{F}^{2} / 2 m^{*}=\epsilon\left(k_{c}\right)\right]$ which assures the validity of the boundary condition for the velocity operator.

\section{Relevant issues and outlook}

Relevant time scales. Typical parameters for a finite-size electron beam splitter (tunnel coupled to reservoirs) defined on a GaAs 2DEG are: a device size $L_{0} \sim 1 \mu \mathrm{m}$, a Fermi velocity in the range $v_{F} \sim 10^{4}-10^{5} \mathrm{~m} / \mathrm{s}$ and an orbital coherence length of $\sim 1 \mu \mathrm{m}$ [21]. These values lead to traversal times $\tau_{t}=L_{0} / v_{F}$ in the range $\sim 10-100 \mathrm{ps}$; these are lower bounds for the actual dwell time $\tau_{\text {dwell }} \sim 1 / \gamma_{R}$ of the electrons in the beam splitter, where $\gamma_{R}$ is the tunnelling rate from the leads of the beam splitter to the reservoirs. Hence the electrons keep their orbital coherence across the beam-splitter at low temperatures. Moreover, long spin dephasing times in semiconductors ( $\sim 100 \mathrm{~ns}$ for bulk GaAs [52]) should allow the propagation of entangled electrons without loss of spin coherence.

For the noise calculation with entangled/unentangled pairs, we have assumed discrete energy levels in the incoming leads. A "particle-in-a-box" estimate of the level spacing $\delta \varepsilon$ due to longitudinal quantization of the beam splitter leads yields $\delta \varepsilon \sim \hbar v_{F} / L_{0} \sim 0.01-0.1 \mathrm{meV}$. The relevant broadening of these levels is given by the coupling $\gamma_{R} \ll \delta \varepsilon$, which justifies the discrete level assumption. Here we take $\gamma_{R} \lesssim \gamma \sim 1 \mu \mathrm{eV}$, where $\gamma$ is the tunnelling 
rate from the entangler to the beam splitter (Sec. 2). In addition, the stationary state description we use requires that the electrons have enough time to "fill in" the extended states in the beam splitter before they leave to the reservoirs: $\tau_{\text {dwell }} \gtrsim \tau_{\text {inj }} \sim 1 / \gamma$. Here $\tau_{\text {inj }}$ is the injection time from the entangler to the beam splitter. To have well separated pairs of entangled electrons, we also need $\tau_{\text {delay }}<\tau_{\text {pairs }} \sim$ ns (Sec. 2$)$, where $\tau_{\text {delay }}(\sim$ ps $)$ is the time delay between two entangled electrons of the same pair, and $\tau_{\text {pairs }}(\sim \mathrm{ns})$ is the time separation between two subsequent pairs.

Interactions in the beam splitter. For entangled electrons it would be advantageous to reduce electron-electron interaction in the beam splitter, which is the main source of orbital decoherence at low temperatures. This could be achieved by depleting the electron sea in the beam splitter, e.g., by using the lowest channel in a quantum point contact. A further possibility is to use a superconductor for the beam splitter [76]. A superconductor would have the advantage that the entangled electrons could be injected into the empty quasiparticle states right at their chemical potential. Because of the large gap $\Delta$ between these states and the condensate, the injected electron cannot exchange energy (nor spin) with the underlying condensate of the superconductor.

An alternative way to detect entangled pairs would be to use a superconductor as an analyzer: arriving entangled (spin-singlet) pairs can enter the superconductor whereas any triplet state is not allowed. Thus, the current of entangled pairs is larger than otherwise.

\section{Noise of a double QD near the Kondo regime}

Spin-flip processes in a spin $1 / 2$ quantum dot attached to leads result in a renormalization of the single-particle transmission coefficient $\mathrm{T}$, giving rise to the Kondo effect [77] below the Kondo temperature $T_{K}$. Theoretical studies on shot noise in this system are available [78]-[80], and show that the noise $S$ obeys qualitatively the same formula as for noninteracting electrons but with a renormalized T. Here, we consider a system where the spin fluctuations (that are enhanced near the Kondo regime) strongly affect the noise, resulting in some cases in super-Poissonian noise - a result which cannot be obtained from the "non-interacting" formula.

We consider two lateral quantum dots (DD), connected in series between two metallic leads via tunnel contacts, see inset of Fig. 12a. The dots are tuned into the Coulomb blockade regime, each dot having a spin $1 / 2$ ground state. The low energy sector of the DD consists of a singlet $|S\rangle$ and a triplet $|T\rangle \equiv$ $\left\{\left|T_{+}\right\rangle,\left|T_{0}\right\rangle,\left|T_{-}\right\rangle\right\}$, with the singlet-triplet splitting $K$. The Kondo effect in this system has been studied extensively [82]-[85]. Two peculiar features in the linear conductance $G$ have been found: a peak in $G$ vs the inter-dot tunnel 
coupling $t_{H}$ (see Fig. 12a), revealing the non-Fermi-liquid critical point of the two-impurity Kondo model (2IKM) [86]; and a peak in $G v s$ an applied perpendicular magnetic field $B$ (see Fig. $12 b$ ), as a result of the singlet-triplet Kondo effect at $K=0$ [82].

The problem of shot noise in DDs with Kondo effect is rather involved. Here we propose a phenomenological approach. For bias $\Delta \mu \gg T_{K}, K$, the scattering problem can be formulated in terms of the following scattering matrix

$$
\begin{aligned}
\mathrm{s}= & \left(\begin{array}{cc}
r_{S} & t_{S} \\
t_{S} & r_{S}
\end{array}\right)|S\rangle\left\langle S\left|+\left(\begin{array}{cc}
r_{T} & t_{T} \\
t_{T} & r_{T}
\end{array}\right)\right| T\right\rangle\langle T| \\
& +\left(\begin{array}{ll}
r_{T S} & t_{T S} \\
t_{T S} & r_{T S}
\end{array}\right)|T\rangle\left\langle S\left|+\left(\begin{array}{ll}
r_{S T} & t_{S T} \\
t_{S T} & r_{S T}
\end{array}\right)\right| S\right\rangle\langle T|,
\end{aligned}
$$

where $t_{i(j)}$ and $r_{i(j)}$ are the transmission and reflection amplitudes. The spin fluctuations in the DD cause fluctuations in the transmission through the DD. The dominant mechanism is qualitatively described by the following stochastic model

$$
f(t)=\left[f_{1}(t)(1-F(t))+f_{2}(t) F(t)\right](1-|\dot{F}(t)|)+f_{3}(t)|\dot{F}(t)|,
$$

where $f_{i}(t)=0,1$ is a white noise $(i=1,2,3)$ with $\left\langle f_{i}(t)\right\rangle=\bar{f}_{i}$ and $\left\langle f_{i}(t) f_{i}(0)\right\rangle-\bar{f}_{i}^{2}=\bar{f}_{i}\left(1-\bar{f}_{i}\right) \delta(t / \Delta t)$, and $F(t)=0,1$ is a telegraph noise with $\bar{F}=\beta /(1+\beta)$ and $\langle F(t) F(0)\rangle-\bar{F}^{2}=\beta \exp (-c t) /(1+\beta)^{2}$, for $t \geq 0$. In this model, the time $t$ is discretized in intervals of $\Delta t=h / 2 \Delta \mu$. The derivative $\dot{F}(t)$ takes values $0, \pm 1$. The function $f_{1(2)}(t)$ describes tunnelling through the DD, with the DD staying in the singlet (triplet) state, while $f_{3}(t)$ describes tunnelling accompanied by the DD transition between singlet and triplet. The relation to formula (42) is given by: $\bar{f}_{1}=\left|t_{S}\right|^{2}=$ $1-\left|r_{S}\right|^{2}, \bar{f}_{2}=\left|t_{T}\right|^{2}=1-\left|r_{T}\right|^{2}$, and $f_{3}=\left|t_{S T}\right|^{2} /\left(\left|t_{S T}\right|^{2}+\left|r_{S T}\right|^{2}\right)=$ $\left|t_{T S}\right|^{2} /\left(\left|t_{T S}\right|^{2}+\left|r_{T S}\right|^{2}\right)$. The telegraph noise is described by two parameters: $\beta=w_{12} / w_{21}$ and $c=w_{12}+w_{21}$, where $w_{i j}$ is the probability to go from $i$ to $j$.

The quantity of interest is the Fano factor $F=S / e|I|$. For a single-channel non-interacting system, one has $F=1-\mathrm{T}$. In order to show the effect of interaction, we introduce the factor $P=F /(1-\mathrm{T})$. The noise power at zero frequency is then given by $S=2 e I_{\mathrm{imp}} \mathrm{T}(1-\mathrm{T}) P$, where $I_{\mathrm{imp}}=2 e \Delta \mu / h$. For the average transmission probability we obtain

$$
\mathrm{T} \equiv\langle f\rangle=\frac{\bar{f}_{1}+\beta \bar{f}_{2}}{1+\beta}+\frac{\beta c \Delta t}{(1+\beta)^{2}}\left(2 \bar{f}_{3}-\bar{f}_{1}-\bar{f}_{2}\right) .
$$

The noise can be calculated as $S=2 e I_{\mathrm{imp}} S_{f}$, with $S_{f}=\mathrm{T}(1-\mathrm{T})+\Delta S_{f}$, where

$$
\Delta S_{f}=\frac{2 \beta}{(1-q)(1+\beta)^{2}}\left\{q\left(\bar{f}_{1}-\bar{f}_{2}\right)^{2}+\frac{c \Delta t\left(\bar{f}_{1}-\bar{f}_{2}\right)}{(1+\beta)} \times\right.
$$



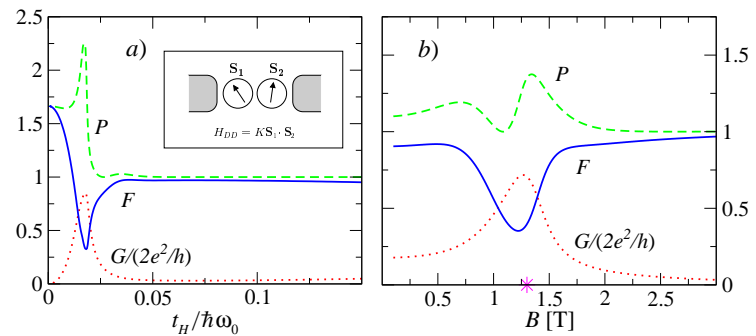

Figure 12. a) Linear conductance $G$ (dotted line), Fano factor (solid line), and the factor $P$ (dashed line), in vicinity of the 2IKM critical point. Inset: DD setup. $b$ ) Similar to ( $a$ ), but in the vicinity of the singlet-triplet Kondo effect (“*” denotes $K=0$ ).

$$
\begin{aligned}
& {\left[\bar{f}_{3}(\beta-1)(q+1)+\bar{f}_{1}(1-\beta q)+\bar{f}_{2}(q-\beta)\right]+} \\
& \left.\frac{(c \Delta t)^{2}}{4}\left[\left(2 \bar{f}_{3}-\bar{f}_{1}-\bar{f}_{2}\right)^{2}-\left(\bar{f}_{1}-\bar{f}_{2}\right)^{2}\right]\right\}
\end{aligned}
$$

with $q=\exp (-c \Delta t)$. The factor $P$ is then given by $P=1+\Delta S_{f} /\left(\mathrm{T}-\mathrm{T}^{2}\right)$. Deviations of $P$ from $P=1$ show the effect of interactions in the DD. We plot the Fano factor and the factor $P$ for a DD on Fig. 12. The results show that the spin fluctuations affect the shot noise in the regions where $K \lesssim T_{K}$. A peculiar feature in $P$ is found both at the 2IKM critical point (Fig. 12a) and at the point of the singlet-triplet Kondo effect (Fig. 12b).

For $\Delta \mu \ll T_{K}$ the DD spin is screened, and correlations between two electrons passing through the DD occur only via virtual excitations of the Kondo state. The shot noise is expected to qualitatively obey the non-interacting formula with the renormalized $\mathrm{T}$.

\section{Summary}

We presented our recent works on shot noise for spin-entangled electrons and spin-polarized currents in novel beam splitter geometries. After a detailed description of various schemes ("entanglers") to produce entangled spin states, we calculated shot noise within the scattering approach for a beam splitter with and without a local s-o interaction in the incoming leads. We find that the s-o interaction significantly alters the noise. Entangled/unentangled pairs and spin-polarized currents show sizable shot noise oscillations as a function of the Rashba phase. Interestingly, we find an additional phase modulation due to s-o induced interband coupling in leads with two channels. Shot noise measurements should allow the identification/characterization of both entangled and unentangled pairs as well as spin-polarized currents. Finally, we find that the s-o coupling constant $\alpha$ is directly related to the Fano factor; this offers an alternative means of extracting $\alpha$ via noise. 
This work was supported by NCCR Nanoscience, the Swiss NSF, DARPA, and ARO.

\section{References}

1. W. Schottky, Ann. Phys. 57 (1918) 541.

2. Ya. M. Blanter and M. Büttiker, Phys. Rep. 336, 1 (2000).

3. D. Loss and E.V. Sukhorukov, Phys. Rev. Lett. 84, 1035 (2000), cond-mat/9907129.

4. G. Burkard, D. Loss, and E.V. Sukhorukov, Phys. Rev. B 61, R16303 (2000), condmat/9906071. For an early account see D. P. DiVincenzo and D. Loss, J. Magn. Magn. Mat. 200, 202 (1999), cond-mat/9901137.

5. W. D. Oliver et al., in Quantum Mesoscopic Phenomena and Mesoscopic Devices in Microelectronics, vol. 559 of NATO ASI Series C: Mathematical and Physical Sciences, eds. I. O. Kulik and R. Ellialtioglu (Kluwer, Dordrecht, 2000), pp. 457-466.

6. F. Taddei and R. Fazio, Phys. Rev. B 65, 075317 (2002).

7. J. C. Egues, G. Burkard, and D. Loss, to appear in the Journal of Superconductivity; cond-mat/0207392.

8. J. C. Egues, G. Burkard, and D. Loss, Phys. Rev. Lett. 89, 176401 (2002); condmat/0204639.

9. B. R. Bulka et al. Phys. Rev. B 60, 12246 (1999).

10. F. G. Brito, J. F. Estanislau, and J. C. Egues, J. Magn. Magn. Mat. 226-230, 457 (2001).

11. F. M. Souza, J. C. Egues, and A. P. Jauho, cond-mat/0209263.

12. J. J. Sakurai, Modern Quantum Mechanics, San Fu Tuan, Ed., (Addison-Wesley, New York, 1994); (Ch. 3, p. 223). See also J. I. Cirac, Nature 413, 375 (2001).

13. Semiconductor Spintronics and Quantum Computation, Eds. D. D. Awschalom, D. Loss, and N. Samarth (Springer, Berlin, 2002).

14. P. Recher, E.V. Sukhorukov, and D. Loss, Phys. Rev. B 63, 165314 (2001); condmat/0009452.

15. D. S. Saraga and D. Loss, cond-mat/0205553.

16. R. Fiederling et al., Nature 402, 787 (1999); Y. Ohno et al., Nature 402, 790 (1999).

17. See J. C. Egues Phys. Rev. Lett. 80, 4578 (1998) and J. C. Egues et al. Phys. Rev. B 64, 195319 (2001) for ballistic spin filtering in semimagnetic heterostructures.

18. P. Recher, E. V. Sukhorukov, and D. Loss, Phys. Rev. Lett. 85, 1962 (2000), condmat/0003089.

19. P. Recher and D. Loss, Phys. Rev. B 65, 165327 (2002), cond-mat/0112298.

20. V.N. Golovach and D. Loss, cond-mat/0109155.

21. R. C. Liu et al., Nature (London), 391, 263 (1998).

22. M. Henny et al., Science 284, 296 (1999); W. D. Oliver et al., Science 284, 299 (1999). See also M. Büttiker, Science 284, 275 (1999).

23. G. Feve et al. (cond-mat/0108021) also investigate transport in a beam splitter configuration. These authors assume a "global" s-o interaction and formulate the scattering approach using Rashba states in single-moded leads.

24. S. Datta and B. Das, Appl. Phys. Lett. 56, 665 (1990).

25. L.P. Kouwenhoven, G. Schön, L.L. Sohn, Mesoscopic Electron Transport, NATO ASI Series E: Applied Sciences-Vol.345, 1997, Kluwer Academic Publishers, Amsterdam.

26. D. Loss and D. P. DiVincenzo, Phys. Rev. A 57, 120 (1998), cond-mat/9701055.

27. M.-S. Choi, C. Bruder, and D. Loss, Phys. Rev. B 62, 13569 (2000); cond-mat/0001011.

28. C. Bena, S. Vishveshwara, L. Balents, and M.P.A. Fisher, Phys. Rev. Lett. 89, 037901 (2002). 
29. G.B. Lesovik, T. Martin, and G. Blatter, Eur. Phys. J. B 24, 287 (2001).

30. R. Mélin, cond-mat/0105073.

31. V. Bouchiat et al., cond-mat/0206005.

32. W.D. Oliver, F. Yamaguchi, and Y. Yamamoto, Phys. Rev. Lett. 88, 037901 (2002).

33. S. Bose and D. Home, Phys. Rev. Lett. 88, 050401 (2002).

34. In principle, an entangler producing entangled triplets $|\uparrow \downarrow\rangle+|\downarrow \uparrow\rangle$ or orbital entanglement would also be desirable.

35. This condition reflects energy conservation in the Andreev tunnelling event from the SC to the two QDs.

36. This reduction factor of the current $I_{2}$ compared to the resonant current $I_{1}$ reflects the energy cost in the virtual states when two electrons tunnel via the same QD into the same Fermi lead and are given by $U$ and/or $\Delta$. Since the lifetime broadenings $\gamma_{1}$ and $\gamma_{2}$ of the two QDs 1 and 2 are small compared to $U$ and $\Delta$ such processes are suppressed.

37. P. Recher and D. Loss, Journal of Superconductivity: Incorporating Novel Magnetism 15 (1): 49-65, February 2002; cond-mat/0205484.

38. A.F. Volkov, P.H.C. Magne, B.J. van Wees, and T.M. Klapwijk, Physica C 242, 261 (1995).

39. M. Kociak, A.Yu. Kasumov, S. Guron, B. Reulet, I.I. Khodos, Yu.B. Gorbatov, V.T. Volkov, L. Vaccarini, and H. Bouchiat, Phys. Rev. Lett. 86, 2416 (2001).

40. M. Bockrath et al., Nature 397, 598 (1999).

41. R. Egger and A. Gogolin, Phys. Rev. Lett. 79, 5082 (1997); R. Egger, Phys. Rev. Lett. 83, 5547 (1999).

42. C. Kane, L. Balents, and M.P.A. Fisher, Phys. Rev. Lett. 79, 5086 (1997).

43. L. Balents and R. Egger, Phys. Rev. B, 64035310 (2001).

44. For a review see e.g. H.J. Schulz, G. Cuniberti, and P. Pieri, cond-mat/9807366; or J. von Delft and H. Schoeller, Annalen der Physik, Vol. 4, 225-305 (1998).

45. The interaction dependent constants $A_{b}$ are of order one for not too strong interaction between electrons in the LL but are decreasing when interaction in the LL-leads is increased [19]. Therefore in the case of substantially strong interaction as it is present in metallic carbon nanotubes, the pre-factors $A_{b}$ can help in addition to suppress $I_{2}$.

46. Since $\gamma_{\rho-}>\gamma_{\rho+}$, it is more probable that two electrons coming from the same Cooper pair travel in the same direction than into different directions when injected into the same LL-lead.

47. In order to have exclusively singlet states as an input for the beamsplitter setup, it is important that the LL-leads return to their spin ground-state after the injected electrons have tunnelled out again into the Fermi leads. For an infinite LL, spin excitations are gapless and therefore an arbitrary small bias voltage $\mu$ between the SC and the Fermi liquids gives rise to spin excitations in the LL. However, for a realistic finite size LL (e.g. a nanotube), spin excitations are gapped on an energy scale $\sim \hbar v_{F} / L$, where $L$ is the length of the LL. Therefore, if $k_{B} T, \mu<\hbar v_{F} / L$ only singlets can leave the LL again to the Fermi leads, since the total spin of the system has to be conserved. For metallic carbon nanotubes, the Fermi velocity is $\sim 10^{6} \mathrm{~m} / \mathrm{s}$, which gives an excitation gap of the order of a few meV for $L \sim \mu \mathrm{m}$; this is large enough for our regime of interest.

48. A singlet-triplet transition for the ground state of a quantum dot can be driven by a magnetic field; see S. Tarucha et al., Phys. Rev. Lett. 84, 2485 (2000).

49. This symmetric setup of the charging energy $U$ is obtained when the gate voltages are tuned such that the total Coulomb charging energies in $D_{C}$ are equal with zero or two electrons.

50. K. Blum, Density Matrix Theory and Applications (Plenum, New York, 1996).

51. T.H. Oosterkamp et al., Nature (London) 395, 873 (1998); T. Fujisawa et al., Science 282, 932 (1998). 
52. J.M. Kikkawa and D.D. Awschalom, Phys. Rev. Lett. 80, 4313 (1998).

53. I. Malajovich, J. M. Kikkawa, D. D. Awschalom, J. J. Berry, and D. D. Awschalom, Phys. Rev. Lett. 84, 1015 (2000); I. Malajovich, J. J. Berry, N. Samarth, and D. D. Awschalom, Nature 411, 770 (2001).

54. M. Johnsson and R. H. Silsbee, Phys. Rev. Lett. 55, 1790 (1985); M. Johnsson and R. H. Silsbee, Phys. Rev. B 37, 5326 (1988); M. Johnsson and R. H. Silsbee, Phys. Rev. B 37, 5712 (1988).

55. F. J. Jedema, A. T. Filip, and B. J. van Wees, Nature 410, 345 (2001); F. J. Jedema, H. B. Heersche, J. J. A. Baselmans, and B. J. van Wees, Nature 416, 713 (2002).

56. In addition, for fully spin-polarized leads the device can act as a single spin memory with read-in and read-out capabilities if the dot is subjected to a ESR source.

57. This is true as long as the Zeeman splitting in the leads is much smaller than their Fermi energies.

58. H.-A. Engel and D. Loss, Phys. Rev. B 65, 195321 (2002), cond-mat/0109470.

59. S. Kawabata, J. Phy. Soc. Jpn. 70, 1210 (2001).

60. N.M. Chtchelkatchev, G. Blatter, G.B. Lesovik, and T. Martin, cond-mat/0112094.

61. M. Büttiker, Phys. Rev. B 46, 12485 (1992); Th. Martin and R. Landauer, Phys. Rev. B 45, 1742 (1992). For a recent comprehensive review on shot noise, see Ref. [2].

62. Our noise definition here differs by a factor of two from that in the review article by Blanter and Büttiker (Ref. [2]); these authors define their power spectral density of the noise with a coefficient two in front (see definition following Eq. (49) and footnote 4 in Ref. [2]). We use a standard Fourier transform (no factor of two in front) to define the noise spectral density.

63. For a discrete energy spectrum we need to insert a density-of-states factor $\nu$ in the current and noise definitions; see Ref. [4].

64. Note that the uncorrelated-beam case here refers to a beam splitter configuration with only one of the incoming leads "open". This is an important point since a beam splitter is noiseless for (unpolarized) uncorrelated beams in both incoming leads.

65. G. Engels et al. Phys. Rev. B 55, R1958 (1997); J. Nitta et al., Phys. Rev. Lett. 78, 1335 (1997); D. Grundler Phys. Rev. Lett. 84, 6074 (2000); Y. sato et al. J. Appl. Phys. 89, 8017 (2001).

66. A. V. Moroz and C. H. W. Barnes, Phys. Rev. B 60, 14272 (1999); F. Mireles and G. Kirczenow, ibid. 64, 024426 (2001); M. Governale and U. Zülicke, Phys. Rev. B 66073311 (2002).

67. G. Lommer et al., Phys. Rev. Lett. 60, 728 (1988), G. L. Chen et al., Phys. Rev. B 47, 4084(R) (1993), E. A. de Andrada e Silva et al., Phys. Rev. B 50, 8523 (1994), and F. G. Pikus and G. E. Pikus Phys. Rev. B 51, 16928 (1995).

68. Yu. A. Bychkov and E. I. Rashba, JETP Lett. 39, 78 (1984).

69. L. W. Molenkamp et al., Phys. Rev. B 64, R121202 (2001); M. H. Larsen et al., ibid. 66, 033304 (2002).

70. The Rashba-active region in lead 1 is (supposed to be) electrostatically induced. This implies that there is no band-gap mismatch between the Rashba region and the adjacent regions in lead 1 due to materials differences. There is, however, a small mismatch arising from the Rashba energy $\epsilon_{R}$; this is the amount the Rashba bands are shifted down with respect to the bands in the absence of s-o orbit in the channel. Since typically $\epsilon_{R} \ll \varepsilon_{F}$, we find that the transmission is indeed very close to unity (see estimate in Ref. [8]).

71. Note that the velocity operator is not diagonal in the presence of the Rashba interaction.

72. J. C. Egues, G. Burkard, and D. Loss, cond-mat/0209692.

73. In the absence of the s-o interaction, we assume the wire has two sets of spin-degenerate parabolic bands for each $k$ vector. In the presence of s-o interaction but neglecting s-o induced interband coupling, there is a one-to-one correspondence between the parabolic 
bands with no spin orbit and the Rashba bands; hence they can both be labelled by the same indices.

74. N. W. Ashcroft and N. D. Mermin, Solid State Physics, Ch. 9. (Holt, Rinehart, and Winston, New York, 1976).

75. G. Schmidt, D. Ferrand, L. W. Molenkamp, A. T. Filip, and B. J. van Wees, Phys. Rev. B 62, R4790 (2000).

76. L. P. Kouwenhoven, private communication.

77. L. I. Glazman and M.E. Raikh, JETP Lett. 47, 452 (1988); T. K. Ng and P. A. Lee, Phys. Rev. Lett. 61, 1768 (1988).

78. Y. Meir and A. Golub, Phys. Rev. Lett. 88, 116802 (2002).

79. F. Yamaguchi and K. Kawamura, Physica B 227, 116 (1996).

80. A. Schiller and S. Hershfield, Phys. Rev. B 58, 14978 (1998).

81. G. Burkard, D. Loss, and D.P. DiVincenzo, Phys. Rev. B 59, 2070 (1999), condmat/9808026.

82. V. N. Golovach and D. Loss, cond-mat/0109155.

83. W. Izumida and O. Sakai, Phys. Rev. B 62, 10260 (2000).

84. A. Georges and Y. Meir, Phys. Rev. Lett. 82, 3508 (1999).

85. T. Aono and M. Eto, Phys. Rev. B 63, 125327 (2001).

86. I. Affleck, A. W. W. Ludwig, and B. A. Jones, Phys. Rev. B 52, 9528 (1995). 
noise_cm.tex; $28 / 10 / 2018 ; 0: 04 ;$ p.36 